\title{
SOZIALE STILE DES MITEINANDER-SPRECHENS. BEOBACHTUNGEN ZU FORMEN DER KONFLIKTBEARBEITUNG IN ZWEI FRAUENGRUPPEN
}

\author{
Inken Keim \\ Johannes Schwitalla
}

\section{EINLEITUNG: ZIELSETZUNG. INFORMATIONEN ZU DEN GRUPPEN}

Im Rahmen des Projekts 'Kommunikation in der Stadt', ' das am Institut für deutsche Sprache durchgeführt wird, untersuchen wir das sprachlich kommunikative Verhalten in zwei Frauengruppen daraufhin, wie sich in der Realisierung bestimmter kommunikativer Muster bei der Bewältigung spezifischer Aufgaben, nämlich der Konfliktbearbeitung, die soziale Identität dieser Gruppen zeigt.

Die erste Frauengruppe, die 'Bastelgruppe', stammt aus einem Stadtteil der Mannheimer Innenstadt mit der volkstümlichen Bezeichnung "die Filsbach". 2 Die Gruppe besteht aus ca. 25 Frauen, die sich seit Jahren regelmäßig wöchentlich im Nebenraum eines gemeinnützigen Vereins treffen. Die meisten von ihnen gehören zur angestammten Stadtteilbevölkerung, vorwiegend Arbeiter, Handwerker und kleinere Geschäftsleute. Die Treffen dienen vor allem der Geselligkeit, auch wenn der äußere Anlaß das Basteln und Handarbeiten ist. Die Treffen werden von einem Vereinsmitglied betreut. Inken Keim war vier Jahre Mitglied dieser Gruppe. Sie konnte in dieser Zeit regelmäßig Tonbandaufnahmen machen.

Die zweite Gruppe, die 'Literaturgruppe', besteht aus neun Frauen, die sich vierzehntägig reihum in der Wohnung einer Teilnehmerin treffen, um sich aus literarischen Erzählungen und Romanen vorzulesen. Diese Frauen wohnen in den bevorzugten Wohnbereichen des Neubaustadtteils Mannheim-Vogelstang. ${ }^{3}$ Sie gehören zur 'tonangebenden' Schicht des Stadtteils. Neun ihrer Treffen wurden von Johannes Schwitalla im Jahr 1985 aufgezeichnet, wobei er nur bei einem selbst dabei war.

Die beiden Frauengruppen sind unter mehreren Aspekten vergleichbar: Es sind Freizeitgruppen, die sich im wesentlichen unterhalten wollen. Die Mitglieder sind Frauen in mittleren bis älteren Lebensjahren, meist verheiratet mit zum Teil schon erwachsenen Kindern. Es sind vor allem Hausfrauen, einige arbeiten auch außer Haus.

Neben diesen Gemeinsamkeiten gibt es aber eine Reihe von Unterschieden, die einen direkten Vergleich des Kommunikationsverhaltens nicht zulassen. Vor allem fol-

1 Zu Anlage und Ziel des Projekts vgl. W. Kallmeyer (1989b).

2 Zur Darstellung dieses Mannheimer Stadtteils: I. Keim (1989).

3 Zur Darstellung des Mannheimer Stadtteils Vogelstang: J. Schwitalla (1989). 
gende Unterschiede zwischen den Gruppen halten wir für wichtig und führen sie hier stichwortartig auf:

BASTELGRUPPE

\section{Zugehörigkeit zu unterschiedlichen sozialen Welten:}

'Einfache' Leute der deutschen Stammbevölkerung aus einem alten, durch Sanierung, hohen Ausländeranteil und Armut charakterisierten Stadtteil.

\section{Unterschiedliche Ziele der Treffen:}

Äußerer Anlaß ist das Bastelangebot eines gemeinnützigen Vereins zur Wiederbelebung des Stadtteils. Das eigentliche Ziel der Treffen ist jedoch Geselligkeit und Unterhaltung (je mehr man lachen konnte, desto schöner war das Treffen).
Angehörige der sozialen tonangebenden Schicht eines neuerbauten Stadtteils, der von der Planung her sozial gemischt ist. Die Frauen oder ihre Männer üben dort organisatorische Tätigkeiten aus in den Kirchen, in der Politik und in Freizeitorganisationen des Sports, der Kunst und der Bildung.

Gemeinsames Lesen und Besprechen von literarisch anspruchsvollen Romanen und Erzählungen; zum Teil konkurrierend dazu das Interesse, sich mit Gleichgesinnten über familiäre Probleme auszutauschen, sich verstanden zu wissen. Regelmäßiges Informieren über familiäre Ereignisse.

\section{Unterschiedliche Organisationsprinzipien der Gruppentreffen:}

Fremdinitiative, die Treffgelegenheit wird vom Verein angeboten; Programmangebote und Materialien dazu werden vom Veranstalter verantwortet. Zur Aufrechterhaltung der Treffen ist wenig Eigeninitiative notwendig und kaum Eigenaufwand zur Durchführung der Treffen; lockere Teilnahmeregelung.
Selbstinitiative und Selbstverantwortlichkeit für das Gelingen und die Aufrechterhaltung der Treffen; impliziter Zwang zur Teilnahme. Hohes $\mathrm{Ma} B$ an Engagement und viel Vorbereitungsaufwand für die Treffen (ausführliche Diskussionen über die zu lesenden Romane).

\section{Unterschiedliche Vertrautheit der Gruppenmitglieder:}

Durch langiähriges Bekanntsein und enges Zusammenwohnen sind die Biographien der Frauen wechselseitig bekannt; hohe Durchsichtigkeit der privaten und sozialen Verhältnisse; gleichzeitig großes Bedürfnis nach Distanz; kaum Einladungen in Wohnungen; Abschirmung des privaten Bereichs vor der Gruppe; enge Vertrautheit nur zwischen einzelnen Mitgliedern der Gruppe, doch ohne deren jeweilige Familien.
Hohe Gemeinsamkeit der Interessen (Literatur, Kunst); Vertrautheit mit dem Wohn- und Lebensstil der anderen, die eine bewußte Öffnung für die anderen voraussetzt. Relativ kurze persönliche Bekanntschaft; gute Kenntnis der gegenseitigen familiären Situation, zum Teil auch enge, freundschaftliche Beziehungen zwischen einigen Frauen und deren Familien (gemeinsame Urlaube, Ausflüge, Theaterbesuche). 
Unterschiedliche Lebensräume außerhalb der Gruppentreffen:

Die meisten Mitglieder haben soziale Kontakte vor allem innerhalb des Stadtteils; geringe überregionale Mobilität; Kontakte mit höheren sozialen Welten vor allem im institutionellen Kontext; Erfahrung als von dene da oben abhängig.
Leitung anderer Freizeitgruppen mit Teilnehmern aus anderen sozialen Milieus; hohe überregionale Mobilität; weiter Freundesund Bekanntenkreis (auch vermittelt durch die Kollegen der Männer).

Wir gehen davon aus, daß es in beiden Gruppen aufgrund der Zugehörigkeit ihrer Mitglieder zu unterschiedlichen sozialen Welten auch unterschiedliche Konzepte von Unterhaltung, unterschiedliche Auffassungen des Imageschutzes und unterschiedliche Vorstellungen darüber gibt, wie bestimmte Themen oder Probleme besprochen werden sollen. Diese auf verschiedenen Ebenen sehr unterschiedlichen Voraussetzungen der Gruppentreffen bedingen u.a.:

- unterschiedliche Kommunikationsformen zur Aufrechterhaltung und Durchführung der Treffen, und - unterschiedliche Regeln für Nähe und Distanz.

Die Bewältigung dieser kommunikativen Aufgaben ist konstitutiv für den sozialen und kommunikativen Stil der jeweiligen Gruppe. Einige der Formen für die Aufrechterhaltung von sozialem Zusammenhalt und für die Regelung von Nähe/Distanz in den beiden Gruppen, die in der Konfliktbearbeitung eine Rolle spielen, fassen wir hier kurz zusammen.

In der Bastelgruppe ist die ernste und ungebrochene Behandlung von persönlichen Problemen nicht erwünscht, da Unterhaltung und Amüsement Priorität haben. Die meisten Mitglieder haben jedoch persönliche Probleme (Alkoholismus, Arbeitslosigkeit, Abhängigkeit von sozialen Einrichtungen), und aufgrund der räumlichen und sozialen Enge wissen die anderen sehr oft von diesen Problemen; d.h. sie besitzen unfreiwillig erworbenes Wissen aus der Privatsphäre der anderen. Für die Behandlung dieses allgemeinen Wissens gilt in der Gruppe, daß die Betroffene selbst ihr Problem mit 'begrenzter Offenheit' präsentiert und in Form und Modalität den in der Gruppe geltenden Regeln für Geselligkeit entsprechend bearbeitet. Bevorzugte Formen dafür sind Witz, Frotzeln und amüsante Erzählungen. Das persönliche Problem wird dabei thematisch auf körperliche bzw. sexuelle Aspekte reduziert (über Eheprobleme z.B. wird unter sexuellem Aspekt gewitzelt). Gleichzeitig jedoch respektieren die Mitglieder das allgemeine Bemühen um die Erhaltung und Abschirmung eines privaten (Rest-)Bereichs. Aus der Perspektive derjenigen, die Probleme, Schwierigkeiten u.ä. hat, ist es wichtig herauszufinden, was die anderen darüber wissen; nur diesen Teil gilt es offenzulegen. Aus der Perspektive der anderen ist es wichtig zu kontrollieren, $\mathrm{da} ß$ die Betroffene die anderen von ihrem Wissen entlastet, indem sie selbst das allen Bekannte thematisiert. Die Problembearbeitung in der Gruppe gleicht einem Balanceakt zwischen 'begrenzter Offenheit' einerseits und der Ausklammerung aus der Gruppeninteraktion andererseits. 
Bei der Bearbeitung von Konflikten, die Gruppennormverletzungen folgen, wird in der Bastelgruppe ähnlich verfahren. Zuerst muß die Normverletzende ihr 'Vergehen' selbst thematisieren, bevor es direkt und explizit in der Gruppe behandelt wird. Hat die Thematisierung durch die Betroffene noch nicht stattgefunden, wird das Kritikobjekt andeutend und indirekt behandelt; emotionale Aspekte des Konflikts dagegen, Ärger, Wut, Enttäuschung u.ä., werden direkt und stark expressiv zum Ausdruck gebracht.

In der Literaturgruppe dagegen ist die Behandlung persönlicher Probleme in ernster, ungebrochener Modalität wesentlicher Bestandteil der Treffen. Die Frauen respektieren es, wenn eine Frau nicht über ihr Problem sprechen will; sie dringen aber in sie, ihr Herz auszuschütten, wenn sie das Gefühl haben, etwas belaste sie. Über Familienprobleme wird nicht ohne Zustimmung der Betroffenen geredet. Andererseits gilt die Regel, andere Frauen nicht zu sehr mit den eigenen Problemen zu belasten. Bestimmte Themen jedoch, wie die Frauen- und besonders die Ehefrauenrolle, zu denen es in der Gruppe unterschiedliche Einstellungen und lebenspraktische Lösungen gibt, werden behutsam behandelt, meist nur angedeutet, selten argumentativ und in ernsthafter Weise. Divergenzen werden durch Anspielungen und nicht-aggressives Frotzeln angedeutet.

Die Frauen praktizieren einen bestimmten Stil des Umgangs miteinander. Sie sind darauf bedacht, die persönliche Sphäre ${ }^{4}$ der jeweils anderen zu achten und ihr "positives Image" ${ }^{15}$ hervorzuheben, wozu sie einige typisierte Ausdrucksmuster von Höflichkeit verwenden. Diese Grundregel läßt negative Wertungen in ihrer Formulierung behandlungsbedürftig werden; sie verbietet grobe und beleidigende Ausdrücke.

Für eine exemplarische Darstellung von Stilmerkmalen halten wir Konfliktbearbeitungen deshalb für erfolgversprechend, weil es bei Störungen des unproblematischen Ablaufs der Interaktion und bei Schwierigkeiten der Beziehungsgestaltung in erhöhtem Maße darauf ankommt, den rechten Ton zu finden und eigene Interessen, verletzte Gefühle und sich widerstreitende Ansichten in einer Weise zu versprachlichen, die den sprachlich kommunikativen Gruppennormen entspricht.

Unter 'Konflikten' verstehen wir ganz allgemein Diskrepanzen zwischen den Mitgliedern der Gruppen hinsichtlich beziehungsbezogener, wertbezogener und wissensbezogener Geltungsansprüche, die sich die Beteiligten wechselseitig verdeutlichen und gegeneinander zu verteidigen oder durchzusetzen versuchen. ${ }^{6}$

Um Konfliktbearbeitungen in diesen beiden Gruppen gegenüberstellen zu können, wählen wir einige vergleichbare Konfliktanlässe aus, die in beiden Gruppen auftreten, und beschreiben dann exemplarisch einige typische Bearbeitungsmuster. Dabei gehen wir von relativ einfachen Konfliktanlässen zu den schwerwiegenderen über. Es sind dies:

- Störungen des aktuellen gemeinsamen Interaktionsziels;

- Konflikte, die daraus entstehen, daß eine Beteiligte ein Thema tabu halten will, die anderen aber das Bedürfnis haben, darüber zu sprechen;

\footnotetext{
4 Vgl. Raible (1987) 146.

5 Vgl. Brown/Levinson (1987) $13 f$.

6 Vgl. Rehbock (1987) 177, Anm. 1.
} 
- Konflikte, die aus grundsätzlichen und für die Beteiligten relevanten unterschiedlichen Wertungssystemen entstehen, und schließlich

- potentiell gruppensprengendes Verhalten.

Dabei wollen wir die typischen Bearbeitungsverfahren in den Gruppen daraufhin betrachten

- wie der Umgang mit persönlicher Nähe und Distanz geregelt ist;

- aufgrund welcher Bedingungen Konflikte verschärft oder abgeschwächt werden;

- ob sich bei der Konfliktbearbeitung für beide Gruppen typische und bevorzugte Realisierungsformen feststellen lassen und ob sie generellen Regeln der Gruppeninteraktion zugeordnet werden können.

\section{KONFLIKTANLÄSSE UND KONFLIKTBEARBEITUNGEN}

\subsection{STÖRUNGEN DES AKTUELLEN INTERAKTIONSZIELS}

In beiden Gruppen können kommunikative Verhaltensweisen von Gruppenmitgliedern, die dem von der Gesamtgruppe zu einem bestimmten Zeitpunkt fokussierten Interaktionsziel entgegenlaufen, Anlässe zu leichteren Konflikten bieten. In den Gruppen werden unterschiedliche Verhaltensweisen als störend empfunden und Verstöße werden unterschiedlich bearbeitet.

\subsubsection{BASTELGRUPPE: BEILEGEN DER STÖRUNG DURCH ORDNUNGSRUFE}

Im Vergleich zur Literaturgruppe verlaufen Treffen in der Bastelgruppe insgesamt turbulenter. Wenn viele Mitglieder anwesend sind, gibt es meist mehrere kleine Gesprächskreise, die jedoch jederzeit aufgebrochen werden können, wenn eines der Mitglieder ein kommunikatives Angebot an die Gruppe macht, das einen hohen Geselligkeitswert verspricht, z.B. ein neuer Witz, ein amüsantes Erlebnis u.ä.. Die meisten der Gruppe wenden sich dann dem interessanten thematischen Angebot zu, mögliche parallel laufende leisere Zweier- oder Dreiergespräche werden nicht als störend betrachtet. Während es den Hauptakteurinnen der Gruppe meist problemlos gelingt, sich durch spezifische Angebotsformulierungen Gehör zu verschaffen, fällt es der Gruppenbetreuerin, Frau Kranz, schwer, ihre thematischen Initiativen in der Gruppe durchzusetzen, vor allem, wenn sie Organisationsaufgaben betreffen (z.B. Planungsüberlegungen zu Festen, Ausflügen u.ä.). Sie erhält oft Unterstützung durch andere Frauen, die mit Ordnungsrufen die anderen zur Disziplin auffordern. Ordnungsrufe gegen kommunikative Disziplinlosigkeit sind im allgemeinen nicht konfliktträchtig; sie werden, nachdem auf sie verbal reagiert wurde, in der Regel befolgt, ohne Mißstimmung hervorgerufen zu haben. Die verbale Reaktion enthält nur in seltenen Fällen, meist jedoch Frau Kranz gegenüber, eine Entschuldigung für die eigene Disziplinlosigkeit. 
Ordnungsrufe und die jeweiligen Reaktionen bestehen meist aus Formeln, ${ }^{7}$ die je nach Situationsanforderung und nach Modalität der Reaktion variieren. Die Verwendung von relativ festen paarigen Formeln für Zurechtweisung und Reaktion darauf erlauben die schnelle und kommunikativ unaufwendige Bearbeitung von momentanen Interaktionsstörungen. Der Formelcharakter ermöglicht eine unpersönliche Art des Tadels; die direkte persönliche Zurechtweisung auf einen konkreten Verstoß mit der inhärenten Gefahr, einen Konflikt auszulösen, kann damit vermieden werden. Die Verwendung von Formeln gestattet es also den Frauen, eine momentane kommunikative Störung effektiv zu bearbeiten und dabei gleichzeitig die persönliche Distanz zwischen Tadelnder und Getadelter aufrechtzuerhalten.

Ordnungsrufe können spielerische Zurechtweisungen sein, auf die die Getadelten ebenfalls spielerisch durch einen Gegenangriff reagieren. So wurde z.B. der Angriff auf das rücksichtslose, laute Dozieren einer Beteiligten in Konkurrenz zu einer von der Gruppe bevorzugten und fokussierten Aktivität durch den ironischen Angriff mensch is des $e$ schlaui fraa von der Getadelten gekontert durch gibt $=s$ wenigschens äni dohin die was weeß.

Ordnungsrufe können auch ernste direkte Zurechtweisungen sein, z.B.:

mensch sei do = mo ruhisch

die entweder durch Eingestehen, z.B.:

ja isch halt moi gosch

oder durch Kontern, wie z.B.:

mensch geb net so $\delta(=a n)$

erwidert werden.

Größere Rücksichtslosigkeit der Störenden einerseits und Hartnäckigkeit der zur Ordnung Rufenden andererseits können aber auch zu kleinen Konflikten führen, die einer aufwendigeren Bearbeitung bedürfen. Auch dazu werden wiederum Formeln eingesetzt. Das folgende Beispiel, in dem die Konfliktverursacherin den Konflikt für sich erfolgreich beenden kann, erhellt darüber hinaus weitere konstitutive Merkmale der Gruppentreffen: Als Frau Kranz (KR) eine Weihnachtsfeier in der Gruppe besprechen will, beginnt Frau Kunz (KU) parallel dazu mit einer derb-amüsanten Erzählung und setzt sich durch. Frau Kunz ist eine der Hauptgruppenunterhalterinnen, die geschätzt ist wegen ihrer Fähigkeit zu Witz, Komik und Groteske. Als Frau Kunz nach dem ersten derb-witzigen Erzählhöhepunkt ihre Erzählung expandieren will, versucht Frau Bart (BA) zu unterbrechen durch die Ermahnung: die fra kranz will was schbresche. Darauf reagiert Frau Kunz nicht und erzählt voller Intensität weiter. Gerade als sie die nächste Pointe präsentieren will, wird sie von Frau Held (HE) unterbrochen: ${ }^{8}$

7 Zum Begriff der Formel, zur Formelbildung und verschiedenen Formeltypen in der Bastelgruppe vgl. W. Kallmeyer/ I. Keim (1986 und 1989a).

8 Die Erläuterungen zu den Transkripten befinden sich im Anhang. 
$1 \mathrm{HE}$ : die redd heid widda fer allet

K: LACHEN

$2 \mathrm{KU}$ : senset ah ja isch bin jo

$3 \mathrm{HE}$ die fra Kranz woll=doch was schbre"sche: nit

$4 \mathrm{KU}$ : ruhisch sach nix mehr vu=mir herd=a nix mehr

K: $\quad$ EINIGE LACHEN

5 HE: nä" so woll=ma des aa nit hawwe nö

K:

AUCH ANDERE PROTESTIEREN

$6 \mathrm{KU}:$ nei"n sch=hab aa moin schdolz

$7 \mathrm{HE}$ :

$8 \mathrm{IN}$ des war ja langweilich fra kunz

$9 \mathrm{HE}:$ jo: $\quad$ mer $\sin$ froh daß se do"

$10 \mathrm{IN}:$ ohne sie geht des net

$11 \mathrm{KR}$ : ne ich würde des/

$12 \mathrm{KU}: \quad$ \#sch=bin do=nit eiern

K: $\quad$ \#LACHEN IN DER STIMME\#

$13 \mathrm{HE}$ : is nit $\uparrow$ daß ma was $z \mathrm{u}$ lache hawwe

14 IN: LACHT

$15 \mathrm{KU}:$ clo":n her†\#

K: ALIE LACHEN

Mit dem formelhaften Tadel für rücksichtsloses Erzählen (Z. 1 im Sinne von: Sie maßt sich das Rederecht für alle an) unterbricht Frau Held Frau Kunzes Erzählung kurz vor der Pointe und zeigt durch den ungewöhnlich harten Eingriff in eine unterhaltsame Aktivität, daß für die Beteiligten die Planungsinitiative von Frau Kranz zu diesem Zeitpunkt Priorität hat vor der derben Spaß versprechenden Erzählung von Frau Kunz. Frau Kunzes zunehmende Rücksichtslosigkeit ahndet Frau Held durch eine grobe Mißachtung von Frau Kunzes Erzählerrolle. Darauf reagiert Frau Kunz kurz und schroff: sense (= Schluß, Ruhe). Frau Held begründet den harten Eingriff dadurch, daß sie Frau Kranz zum Rederecht verhelfen wollte (Z. 3). Darauf folgt keine Entschuldigung u.ä. von Frau Kunz für die getadelte Rücksichtslosigkeit, sondern sie kündigt in beleidigtem Ton ihre Absicht an, fortan absolut zu schweigen (Z. 4). Auf den fallgebundenen Vorwurf reagiert sie durch Generalisierung ihrer Absicht zur Verhaltensänderung (= schweigen) und bringt sie damit in direkten Zusammenhang mit ihrer Rolle als Unterhalterin; ihre Reaktion enthält so die implizite Drohung, die Unterhalterrolle aufzugeben. Damit evoziert sie Reparaturversu- 
che der anderen (Z. 10). Das Reparaturangebot wehrt sie zunächst noch in 'schmollendbeleidigtem' Ton ab, signalisiert durch die Verwendung der Formel sch=hab aa moin schdolz jedoch, daß ihr Beleidigtsein spielerische Elemente hat.

Nach der expliziten und ernst gemeinten Anerkennung ihrer Rolle als Gruppenunterhalterin durch Frau Held (Z. 9/13) protestiert sie spielerisch gegen die zugeschriebene Rolle, indem sie sie als Clownrolle zurückweist (Z. 15). Doch sie stimmt in das Lachen der übrigen ein und übernimmt anschließend die Rolle.

Frau Kunz kann den Konflikt, den sie durch rücksichtsloses Verhalten auslöste, zu ihrem Vorteil beenden, d.h. nicht sie muß sich bei Frau Kranz entschuldigen, sondern die Tadelnden reparieren ihren Vorwurf:

- weil Frau Kunz die fallbezogen erwünschte Reaktion auf eine Entgleisung in direkten Zusammenhang bringt mit einer für die Gruppentreffen wichtigen Interaktionsform: amüsante Erzählungen mit hohem Unterhaltungswert;

- weil sie weiß, daß sie für diese Interaktionsform ebenso wie für andere Unterhaltungsformen eine der zentralen Figuren der Gruppe ist; und

- weil die momentane Entgleisung ihr in Ausübung gerade dieser Unterhalterrolle passierte.

Die Reparaturen des Vorwurfs durch die anderen bestätigen die Priorität der Unterhalterrolle, der auch gröbere interaktive Entgleisungen nachgeordnet werden. Gelingt bei Entgleisungen der spielerisch-gebrochene Bezug zur Unterhalterrolle, sind Entschuldigungen auch der Betreuerin gegenüber nicht erforderlich; dagegen sind Reparaturen für den Tadel enwartbar.

\subsubsection{LITERATURGRUPPE: IRONISCHE UND SCHERZHAFTE MODALISIERUNGEN ZUR BEILEGUNG DER STÖRUNG}

Die Frauen der Literaturgruppe erheben für sich den Anspruch, an einem Gespräch teilzunehmen, das Auseinanderbrechen in mehrere Gesprächsfoki lehnen sie ab. Die Frauen müssen jedoch beständig mit dem Widerspruch fertig werden, daß sie zwar zusammenkommen, um ein Thema aus der Literatur gemeinsam zu behandeln, daß sie aber andererseits das Bedürfnis haben, bestimmte private Themen entweder allen Anwesenden oder nur bestimmten Adressatinnen mitzuteilen und mit ihnen zu besprechen.

Da dieser Konflikttyp strukturell aus dem Widerstreit zwischen offiziellem Interaktionsziel und eigenen Gesprächsbedürfnissen der Beteiligten vorgegeben ist und häufig vorkommt, haben die Frauen mehr Bearbeitungsmöglichkeiten im Vergleich zu anderen Konflikttypen entwickelt. Bei der Durchführung zeigt sich, daß die Frauen darauf bedacht sind, die persönliche Distanz zu wahren und keine Mißstimmung aufkommen zu lassen. Dies gelingt den Frauen durch die Realisierung ironischer Sprechakte und durch stilistische Abwandlungen und Formulierungsbearbeitungen der Ordnungsrufe. 
a) Ironische Zurechtweisung

Z.B. sagt eine Vorleserin, während sich zwei andere Frauen über einen Pullover unterhalten, der in einer Zeitschrift abgebildet ist: ich hoffe, es stört euch nicht, daß ich lese während ihr die zeitschrift anguckt. Die Ironie entsteht aus der Umkehrung der Agens-Rolle, wer wen stört.

b) Kurze Ordnungsrufe

Ein dringlich gestalteter Ordnungsruf (Imperativ, lauteres Sprechen) wird spielerisch abgeschwächt durch eine Variation zum dialektalen Sprechen:

$1 \mathrm{KE}$ : ja dann müßt=er jetzt a"lle mal zuhören *

2 ST: ja† ich hö:re $\downarrow$

3 EB: <also jetzt laßt emol Erika was sage und seid kurzerhand alle still

Frau Ebert (EB) spricht hier stärker Dialekt, als sie es sonst in ihren Redebeiträgen tut. Eine ähnlich variierte Aufforderung bringen beide Sprecherinnen im folgenden Beispiel:

$1 \mathrm{KE}: \rightarrow$ sol ich bitte um aufmerksamkeit

2 EB: jetz isch aber ruhe $\downarrow$

Hier kontrastieren formelhafte Wendungen aus dem Bereich der Öffentlichkeit und dem eher privaten, vertrauten Umgang zwischen Eltern und Kindern (oder Lehrer - Schüler).

c) Scherzhafte, übertiebene Aufforderung

Damit werden zwei kommunikative Handlungen gleichzeitig vollzogen: einerseits werden durch den gespielten, scherzhaften Charakter die Wirkungspotenzen nicht-modalisierter Sprechakte aufgehoben; andererseits aber wird durch den propositionalen Gehalt der Äußerung dennoch auf kritische Punkte des Verhaltens einer Adressatin verwiesen. Sprachliche Mittel zum Ausdruck der Ironie sind dabei meistens übertreibende Lexik und ein übertrieben empörter, ernster Sprechton. Als Beispiel seien zwei kurze Passagen zitiert, die während der Vorlesephase geäußert wurden, bevor das letzte Beispiel einsetzte. Frau Funke (FU) ist die Vorleserin:

1 FU: bitte $\uparrow \quad$ wa"s war $\uparrow$

2 NW: PRUSTET

3 EB: diese u"nqualifizierten (...)

4 BA: laß dich nicht so ablenkent

$5 \mathrm{SE}$ ich hab gesagt irgendwie is ro:sa und pink

6 BA: \#Gu"drun hö: $r$ mal zu: das (gehort?aeht) nicht

K: $\quad$ \#GESPIEIT EMPÖRT\#

$7 \mathrm{SE}:$ modern he he

$8 \mathrm{NW}$ ha ha ha

$9 \mathrm{KE}$ :

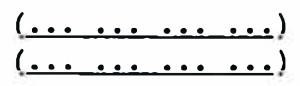


$10 \mathrm{BA}$ : zur sa"che\#

$[\ldots]$

11 GR: nee nee Gudrun ich hab gekuckt hier du hast dich auch mit deinem

12 GR: strickzeug beschäftigt-

13 OT:

STÖHNT\# is ne fre"chheit\# * hier wird einem

$\mathrm{K}:$

\# GESPIELT EMPÖRT \#

14 or: unterste"llt\# \#ob man hier zuhört\# \#oder nicht\#

K: \# SCHMUNZEIND \# \# LACHEND \#

$15 \mathrm{NW}:$

ha ha ha

16 EB:

nei:n des wird

17 EB: doch nich unterste"lit sondern einfach be*o:b*achtet $\downarrow$

18 GR:

des war nur

19 GR: ne feststellung

20 EB: \#ja $\rightarrow$ ganz genau \#

K: \# GESPIELT SCHNIPPISCH \#

Die Modalisierung des Tadels setzt nach dem etwas verschämt gesprochenen Eingeständnis von Frau Senft (SE) ein, die zugibt, über etwas gesprochen zu haben, was nicht zum Thema der vorgelesenen Erzählung gehört. Die Zurechtweisung von Frau Bareis (BA): Gudrun hör mal zu, des gehört nicht zur sache ist wie ein tadelnder Verweis in der Schule. Die Übertreibungen liegen einerseits in der Syntax (Imperativ), vor allem aber in der Prosodie: rhythmisch betonte oder gelängte Vokale: Gu"drun hö:r mal zu: das (gehört?geht) nicht zur sa"che.

Normalerweise wird ein Tadel akzeptiert. Wenn er bearbeitet wird, dann auf spielerische Weise wie hier, wo sich die Störenden (Frau Senft und Frau Ott) ihrer Schuld bewußt sind. Die spielerische Bearbeitung verschiebt das Problem in eine andere Modalität, die gleichzeitig weniger Image-bedrohend ist. Es folgt keine Rechtfertigung der Betroffenen, sondern ein Eingeständnis (Frau Senft Z. 5) bzw. eine gespielte Entrüstung von Frau Ott (OT): is ja ne frechheit ... (Z. 13-14). Auch sie rhythmisiert ihre ersten drei Teilsätze und sie artikuliert das " $t$ " in unterstellt mit einer besonders starken fortis-Aussprache. Sie kann aber diese entrüstete Sprechweise nicht durchhalten, sondern fällt in ein lachendes Sprechen, das vollends ihre Rede als uneigentlich kennzeichnet. Bemerkenswert ist auch, daß Frau Ebert, die eine nachträgliche metakommunikative Definition der Äußerung von Frau Gries (GR) gibt (des wird doch nicht unterstellt, sondern einfach beobachtet), im Wort beobachtet durch zweimalige Pausensetzung den Rhythmus von Frau Ott aufnimmt. Zur größeren Sicherheit folgt abschließend auf die Modalisierung eine abschwächende metakommunikative Äußerung.

d) Rechtfertigung aufgrund der Gruppennormen: 'zur Sache sprechen'

In Beispiel c) Zeile 6/10 wurde auf eine Gruppennorm verwiesen: Die Lektüre darf nur unterbrochen werden, wenn es der Klärung des Vorgelesenen dient (das (geht?gehört) 
nicht zur sache). Nebengespräche, die im Einklang mit dem offiziellen Interaktionsziel stehen, werden geduldet und eine Frau, die deswegen getadelt wird, kann sich unter Berufung auf diese Gruppennorm rechtfertigen.

Im folgenden Beispiel hatte Frau Funke etwas zum vorgelesenen Text nachgefragt; Frau Kerk (KE) ruft die Gruppe zur Ordnung :

$1 \mathrm{KE}$ : \#aber ich glaube wir müssen da wi"rklich mal wenichstens zwanzich

K: \# LEICHT VORWURFSVOLL \#

$2 \mathrm{KE}$ : mimuten hi"ntereinander ohne jeqliche unterbrechuna reden $\downarrow \#$

3 or: $\quad<i a$ du schwätzt ia andauernd dazwi"schen 1

$4 \mathrm{FU}:$ aberk

5 FU: $\rightarrow$ ja aber jetz wu/ des war jetzt wirklich sa"che sonst wär ich

6 FU: überhaupt nich mehr mi"tgekonmen ich hätt eben a"bgeschnallt weil

7 FU: ich nich mehr kapie"rt hab um was es geht-

8 BA: ja $\star \star$

9 or: \#>als ob es mir be/ \#

K: \# GANZ LEISE GEFLÜSTERT \#

$10 \mathrm{KE}: \quad$ und wieder reinkommt $\downarrow$ ** denn es is

$11 \mathrm{KE}$ : eigentlich zau"berhaft geschrieben

12 ST: ja des is (so ironisch) es ist sehr gei:strei:ch- und

$13 \mathrm{NW}$ (schöne) qeschichte

14 FU: $\quad$ ich denke es is nämlich auch au"fschlußreich

$15 \mathrm{KE}: \quad \quad$ seh"r genau beobachtet

16 FU:

17 ST:

es is mal wieder eine der geschichten die man

$18 \mathrm{ST}$ : (nich für sich selber liest t)

19 OT: aber nich extra kochen \# Gerda \# net

K: \# = FRAU FUNKE' \#

Vorwurf und Gegenvorwurf zwischen Frau Kerk und Frau Ott werden von beiden nicht weiter behandelt. Stattdessen rechtfertigt Frau Funke ihre Unterbrechung und begründet sie (Z. 5-7). Eine Replik auf diese Rechtfertigung spricht Frau Ott ganz leise, kaum hörbar, wie für sich selbst gesprochen und dadurch ihrer kommunikativen Qualität entkleidet (Z. 9). Von nun an kooperieren fast alle Beteiligten an der Konfliktbeendigung: Frau Kerk, die Urheberin der Turbulenz, akzeptiert die Rechtfertigung von Frau Funke, indem sie eine - wenn auch deiktisch von ich zu man verschobene - Ergänzung dieser Rechtfertigung gibt (Z. 10-11). Dann folgt ein Themawechsel zu einem Lob des Romans, also von einer negativ zu einer positiv gerichteten Tätigkeit, und in dieses Lob fallen drei weitere Sprecherinnen ein, welche zusätzliche Qualitäten des Romans thematisieren ( $\mathrm{Z}$. 12-18). Die Konfliktphase löst sich also in einem allgemeinen Konsens auf. $\mathrm{Da} B$ der Konflikt keine Beziehungsschädigung in der Gruppe hinterlassen hat, sieht man auch daran, 
daß Frau Ott, die den Konflikt verschärfte (Z. 3), die in der Gruppe übliche Verhaltensregel für Gäste einhält und die Gastgeberin bittet, sich möglichst wenig Mühe mit den Gästen zu machen.

In beiden Gruppen werden also Störungen daran gemessen, wie sehr sie das gemeinsam akzeptierte und für wichtig gehaltene Interaktionsziel der Gruppentreffen behindern. 'Störungen', die diesen Interaktionszielen entsprechen, können in der Literaturgruppe gerechtfertigt werden. Ordnungsrufe werden in beiden Gruppen unterschiedlich durchgeführt, vornehmlich formelhaft und ohne Notwendigkeit zur weiteren verbalen Bearbeitung in der Bastelgruppe; vornehmlich ironisch und scherzhaft eingekleidet, zur Vermeidung eines zu direkten Tadels in der Literaturgruppe.

\subsection{DAS TABUHALTEN VON THEMEN}

Hier behandeln wir Konflikte, die dadurch entstehen, daß Beteiligte sich bei der Behandlung von Themen, die persönliche Probleme tangieren, zurückhalten, bei denen sie sich aber nach der Meinung anderer (der ganzen Gruppe oder einzelner Mitglieder) aktiv beteiligen sollten, und zwar in der Weise, in der Problembehandlungen in den Gruppen geregelt sind.

\subsubsection{BASTELGRUPPE: AUFHEBUNG VON TABU-THEMEN}

Private Probleme werden in der Bastelgruppe nur in einem Bearbeitungsmodus behandelt, der für die anderen Unterhaltungswert hat. Zu den Interaktionsformen mit hohem Unterhaltungswert gehören das Erzählen von sexuellen Witzen, das Erzählen amüsanter derber Geschichten und frotzelnde Phantasie- und Unsinnspiele mit sexueller bzw. allgemein körperlicher Thematik. Unter dem Unterhaltungsaspekt werden ernste persönliche oder familiäre Probleme bei der Präsentation in der Gruppe auf allgemein körperliche bzw. sexuelle Aspekte reduziert und in scherzhafter Modalität als etwas Amüsantes geschildert oder durch Frotzelspiele u.ä. bearbeitet. Außerdem gehört es, wie oben bereits ausgeführt, zu den Regeln der Gruppe, daß man persönliche Probleme, von denen man annimmt oder weiß, daß die anderen sie kennen, in der Gruppe thematisiert, und zwar in amüsanter Form.

Gegen diese Norm verstößt eine der Frauen, die versucht, ihr Problem, das alle kennen, tabu zu halten. Der Verstoß wird indirekt durch 'hartes Frotzeln'9 mit dem Ziel der Bloßstellung des Opfers bestraft. Reagiert das Opfer betroffen, ist das Ziel erreicht; es folgen keine Renormalisierungsaktivitäten der Angreiferin/nen, sondern diese zeigt bzw. zeigen Schadenfreude und Lust an der Rache durch kreischendes Lachen. Zu Inhalt und Verlauf des harten Frotzelns als indirekte Kritik bei Verletzung von Gruppennormen folgendes Beispiel:

9 Zu verschiedenen Typen des Frotzelns vgl. I. Keim (1988). Inhalt, Form und Funktion des Frotzelns in der Bastelgruppe (masch.). 
Die Gruppe unternimmt einen Ausflug in ein Waldgebiet mit Tiergehegen am Rande von Mannheim. Vor dem Wildschweingehege zeigt sich Frau Held (HE) spielerisch übertreibend an einem der Keiler interessiert. Darauf startet Frau Schumann (SU) ihren ersten Angriff auf Frau Held:

1 SU: tdie frau Held is auf den keiler so wild-

$2 \mathrm{IN}$ :

LACHT

3 HE:

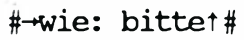

$\mathrm{K}:$

\# SCHEINENTRUSTET \#

4 SU: \#-die sammelt immer männer ${ }^{\Uparrow} \#$

K: \# SCHMUNZEIND \#

$5 \mathrm{HE}$ :

$6 \mathrm{IN}$ :

IACHT do vorne is äna der werd/

$\mathrm{K}:$

$7 \mathrm{HU}:$

$\underline{\text { LACHT }}$

\# sie" \#

\# HEID \#

8 SU: $\quad$ also wenn die ni"x sieht- mä"nner sieht se-

$9 \mathrm{HE}$ : \#wadd emo wennfa doher kummt ${ }^{\text {\# }}$

K: \# ANZÜGLICHES ANDEUTEND \#

lo $\mathrm{HU}$ :

LACGT GRFLL

$11 \mathrm{HJ}: \quad$ des is/ des is so si"tte bei

$12 \mathrm{HE}$ : mir gehts=s wie eisch aa gell $\uparrow$ isch brauch/

$\mathrm{K}$ : HELIES LACHEN

$13 \mathrm{HJ}$ : denne im haus-

$14 \mathrm{HE}: \quad$ \# i"sch brauch kän monn mehrł\#

K: $\quad$ \# BETROFFEN, TIEFE STIMME \#

K: GRELLES LACHEN

$15 \mathrm{HJ}: \quad \quad$ \#des ligat in de verwandtschaft\# LACHT

K: \# HOHE STIMME \#

$16 \mathrm{HE}:$ \#oh liewer gottı\#

$\mathrm{K}:$ \# ABWERTEND \#

17 Kö: wer wee: $\beta$ wer wee: $\beta$

18 SU: \#isch habb schon für zwei bekannte annonciert\#

K: \# BEGINNT NEUES THEMA IM HINTERGRUND \#

$19 \mathrm{HE}$ :

$>$ mit denne haww $=$ isch nix zu due $\downarrow$

$20 \mathrm{HE}$ : isch bin fer misch $\downarrow$

K: $\quad$ THEMENWECHSEL (ANNONCE FÜR BEKANNTE)

Der erste Zug ist sowohl prosodisch als auch auf der Ebene der Handlungszuschreibung als Spiel markiert (hintergründiger Ton, größere Tonhöhenunterschiede bei keiler und wild). Die Formulierung, auf ein männliches Tier wild sein, gehört zu den in der Gruppe üblichen übertreibenden Formen im Spiel, wobei männliche Tiere als Metapher für Män- 
ner fungieren. Frau Schumann charakterisiert damit die spielerische Selbstdarstellung von Frau Held vor dem Wildschweingehege. Frau Held ratifiziert durch ihre in spielerischer Weise übertriebene Entrüstung (tiefere Stimme, langsame Sprechweise, Dehnung des Fragepronomens, Akzent auf jedem Wort, steigende Intonationskontur) und mit dem anschließenden Lachen das Frotzelspiel.

Der zweite Angriff von Frau Schumann die sammelt immer männer ist eine deutliche Steigerung gegenüber dem ersten Angriff: War das Objekt vorher metaphorisch ausgedrückt (Keiler), wird es jetzt direkt genannt (Männer). Die Handlungszuschreibung hat die Form einer empirischen Generalisierung; sie drückt die Verallgemeinerung einer Handlungsweise aus, die im ersten Angriff singulär und indexikal dargestellt wurde. Die soziale Kategorie, auf die im zweiten Angriff verwiesen wird, auf eine Frau 'die viele Männer hat', ist in der Welt der Beteiligten deutlich negativ bewertet.

In der zweiten Reaktion (Z. 5-9) übernimmt Frau Held die ihr zugeschriebene Kategorie dadurch, daß sie in einem prospektiven Phantasiespiel andeutet, wie sich ihrer Meinung nach eine derart kategorisierte Frau verhält; einen zufällig vorbeigehenden Spaziergänger legt sie als Kandidaten fest, den sie anzumachen gedenkt.

Der dritte Angriff von Frau Schumann also wenn die nix sieht, männer sieht se stellt die projizierte spielerische Selbstdarstellung von Frau Helds Verhalten in Form einer Regel dar, die die allgemeine Gültigkeit von Frau Helds Interessen an Männern ausdrückt.

In der dritten Reaktion (Z. 12) nimmt Frau Held Frau Schumanns Regel als für sich selbst geltend auf, subsumiert durch die Partikel aa (auch) sowohl ihren eigenen Fall als auch durch den Vergleich (wie eisch) die anderen unter die soziale Kategorie einer Frau, die nur männer sieht. Dadurch stellt sie eine Gemeinsamkeit zwischen der Angreiferin, sich selbst und dem Publikum her. Ihr Weiterspiel wird unterbrochen durch die parallele Initiative von Frau Hild.

Bis hierher waren Frau Schumann, die Angreiferin, und Frau Held, das 'Opfer', auf der prosodischen Ebene genau aufeinander bezogen und stimmten in Tonhöhenbewegung, Tempo und Rhythmus überein. Angriff und Reaktion waren so auch prosodisch als spielerische Einheiten gekennzeichnet. Bis hierher konnte Frau Held mitspielen. Die Angriffe waren auf sie selbst gerichtet und sie konnte sie interpretieren als auf eine von ihr selbst im Spiel initiierte soziale Kategorie bezogen, auf eine Frau 'mit vielen Männern', eine Kategorie, die in der Realität nicht auf sie zutrifft.

Mit dem vierten Angriff, den jetzt Frau Hild startet: des is/des is so sitte bei denne im haus, verändert sich die Qualität des Spiels. Die von Frau Schumann formulierte Regel (ausschließlich Männer sehen) wird jetzt auf die Wohnumgebung von Frau Held (ein Mehrfamilienhaus) bezogen und erhält dadurch Realitätsgehalt (s.u.). Die Reaktion der Beteiligten ist grelles Lachen.

Frau Held reagiert ernst und betroffen: $i$ "sch brauch kän monn mehr oh liewer gott (tiefe Stimme, abfallende Intonationskontur und deutlich abwertender Ton in oh liewer gott). In ihrer Reaktion weist Frau Held in ernster Modalität die Zuschreibungen zurück, die sie vorher im Spiel selbst inszeniert bzw. übernommen hatte. Nach Frau Helds Betroffenheitsbekundung setzt Frau Hild mit einem weiteren Angriff nach: des liggt in de verwandtschaft, der in Bezug auf den Referenzbereich durch Einschränkung eine Steigerung gegenüber dem vierten Angriff darstellt: Die angeführte Verhaltensregel (ausschließlich 
Männer sehen) trifft nicht auf das gesamte Wohnumfeld von Frau Held zu, sondern nur auf ihre Familie. Zu der räumlichen Nähe (Wohnbereich) kommt jetzt auch die soziale Nähe (Familie). Auf den fünften Angriff zeigt Frau Held wieder Betroffenheit; sie spricht leise und distanziert sich jetzt explizit von ihrer Familie: mit denne haww=isch nix zu due. Zwischen dem fünften Angriff und der fünften Reaktion führt Frau Schumann ein neues Thema ein, wobei sie an einen bereits früher etablierten thematischen Rahmen wieder anknüpft. D.h. Frau Schumann wechselt die Interaktionsform, und nach einer kurzen Pause folgen ihr die anderen.

Um den Fragen nachzugehen, wieso Frau Hilds Angriff Frau Held betroffen macht und sie ihn aufrechterhalten kann, ist zunächst Frau Helds Problem zu erhellen, auf das hier angespielt wird: Es handelt sich um die Nichten von Frau Held, die in demselben Haus wohnen wie sie. Deren Verhalten verletzt eine Reihe von Normen, die in der sozialen Welt der Beteiligten gelten: Sie lassen sich von Männern aushalten und leben vom Sozialamt. Frau Helds Nichten sind mehrmals Gegenstand von ausführlichem Tratsch in der Gruppe. Dabei wird auch explizit Frau Helds nachsichtiges Verhalten den Abweichungen ihrer Nichten gegenüber negativ bewertet, ihr wegen ihrer Nachsichtigkeit eine Mitschuld an der Liederlichkeit der Nichten zugeschrieben.

Frau Hilds fünfter Angriff verweist nun genau auf diese Zusammenhänge. Sie verwendet dafür die allgemein übliche Redewendung: des liggt in de verwandtschaft, die die verhaltensmäßige Ähnlichkeit zwischen Familienmitgliedern thematisiert. Die dem vierten und fünften Angriff zugrundeliegenden Kategorien haben durch den Referenzwechsel auf Frau Helds Nichten jetzt Realitätsgehalt.

Frau Hild stellt hier eine Beziehung zwischen dem spielerisch dargestellten Verhalten von Frau Held (Männer sammeln), dem 'wahren' Verhalten der Nichten und dem 'wahren' ( = nachsichtigen und damit mitschuldigen) Verhalten von Frau Held her. Sie zeigt damit, daß sie den sensiblen Punkt und das Problem im gegenwärtigen Leben von Frau Held kennt. Die übrigen Frauen zeigen ihr Wissen durch schadenfreudiges Lachen.

Frau Held hatte bisher ihr Problem in der Gruppe noch nicht behandelt. Diesbezügliche Andeutungen der anderen überging sie, sie wollte ihr Problem zum Tabuthema machen, obwohl alle davon wußten. Für diesen Zwang zur Geheimhaltung wird sie hier von den anderen durch Bloßstellung bestraft. Außerdem beging sie in der geschilderten Situation einen zusätzlichen Fehler: Vor Beginn der Frotzel-Sequenz, und diese erst auslösend, stellte sie sich selbst spielerisch übertrieben als wild auf Männer dar. In ihrer Inszenierung, die zwar keine Selbst-Referenz in der Realität hat, aber auf ihre Nichten zutrifft, hat sie sich sehr nahe an ihr Tabuthema heranbegeben und schaffte damit selbst den $A n l a ß$ für die Frotzelsequenz. Auf dem Hintergrund dieses Wissens kann jetzt Frau Schumanns erster Zug als Angriff auf Frau Helds übertriebene spielerische Selbstdarstellung verstanden werden, die zu nah an ihrem Problem lag; und Frau Hild bestraft Frau Held sofort für ihr provokant inkonsequentes Verhalten. 


\subsubsection{DIE LITERATURGRUPPE: VORSICHTIGES UMGEHEN MIT TABUTHEMEN}

Die Frauen der Literaturgruppe haben unterschiedliche Auffassungen darüber, ob bestimmte Themen zu einem nicht besprechbaren Intimbereich der einzelnen Mitglieder gehören oder nicht. Diese Frage wird regelmäßig dann thematisiert, wenn es darum geht, ob man Informationen zu möglicherweise schweren Krankheiten von Gruppenmitgliedern oder deren Männern weitergeben darf. Zu umstrittenen, persönlich tangierenden und deshalb potentiell Image-gefährdenden Themen gehören neben schweren Krankheiten auch Konflikte mit den Ehemännern, die Auffassungen über die Rolle als Ehepartner, das Frauenbild allgemein und unterschiedliche finanzielle Ressourcen. Oft werden Gesprächsinhalte, die in diese Richtung gehen, fallengelassen oder aber, wenn der Neuigkeitswert sehr groß ist (z.B. beim Verdacht auf eine schwere Krankheit), erst nach einer metakommunikativen Debatte unter vielen Signalisierungen innerer Hemmungen und der Rücksichtnahme auf die Betroffene besprochen: ich weiß nicht, ob ich dir $=s$ sagen darf; ich wei $\beta$ es auch, aber ich sag =s nich; sag =s lieber nich + LACHEN; ich weiß es nich, ob ich es sagen kann usw. Diese beiden Behandlungsformen entsprechen der Gruppenregel, private Themen mit einem großen Potential an Imagegefährdung nur dann zu besprechen, wenn die Betroffene anwesend ist und von sich aus das Thema einbringt, bzw. in den 'sanften Zwang' einwilligt, ein sie belastendes Problem, das sie angedeutet hat, offen und in ernster Modalität zu besprechen.

Im Gegensatz zur Bastelgruppe wird das Zurückhalten bestimmter Informationen über Lebensumstände und konkrete Ereignisse nicht geahndet. Doch führt es ebenfalls zu Konflikten, wenn eine Frau nicht in derselben Weise offen über familiäre Probleme spricht wie die anderen. Die Durchführungsweise der Konfliktbearbeitung ist dann ein argumentativer Disput, bei dem zwar der zugrundeliegende Vorwurf nicht offen ausgesprochen wird, jedoch die Betroffene sich mit Meinungen oder Deutungen auseinandersetzen muß, die eine mögliche Gefährdung ihres Ansehens, wie sie es vor der Gruppe handhabt, enthalten.

Dazu ein Beispiel, das seiner Länge wegen nicht ganz zitiert werden kann: Mehrere Gruppenmitglieder glauben, daß Frau Senft bemüht ist, von sich und ihrer Familie ein betont harmonisches Bild zu geben. Als sie nun von einer Krankheit spricht, wird sie von Frau Funke und anderen Frauen mit der Meinung konfrontiert, diese Krankheit habe psychische Ursachen. Nach dem Wissen aller Beteiligten würde dies für Frau Senft bedeuten, $\mathrm{da} ß$ sie sich mit Problemen auseinandersetzen müßte, die sie vor den anderen und wahrscheinlich vor sich selbst versteckt. Frau Senft wehrt sich heftig gegen die These der anderen (de"s $i$ "s nich psychisch). Die Opponentin beharrt auf ihrer Meinung: aber warum weist $=e$ des denn von der hand? [...] ich denke, das is ne psychosomatische geschichte. Eine Dritte bietet einen inhaltlichen Kompromiß an: nei:n nicht au"sschließlich, aber da ist doch sicherlich ein faktor dabei. Die Betroffene argumentiert, daß sie fünf Wochen im Urlaub war, wo sie keine Beschwerden hatte, also - so läßt sie ihre Zuhörerinnen schließen müsse ein auf ihre Wohnung lokalisierbarer materieller Stoff ihre Krankheit verursachen. Aber ihre Opponentin kehrt das Argument um; sie wird dabei von Frau Ott und Frau Bareis unterstützt: 
$1 \mathrm{FU}: \# \rightarrow$ ja aber des spricht doch überhaupt nich dagegen daß es

K: \# SEHR SCHNELL, MEHRERE HOCHSCHUÜSSE, UM VERSTÄNDNIS WERBEND \#

2 FU: psychosoma"tisch is im gegentei: $1 \uparrow$

so ne so ne *

3 BA:

$\rightarrow$ ja das würd ich auch so sehn $\downarrow$

4 FU: u"rlaubssituation mi"t deiner familie oder auch ga"nz alleinet * so

$5 \mathrm{FU}$ : was ga"nz anderes wie hie: $r \uparrow$ mit all den-\# $\star \star$ proble:men oder

$6 \mathrm{BA}: \quad j a: \uparrow$

$7 \mathrm{FU}$ : mit all derl/ wei"ß ja nich wahrscheinlich weißt=e se"lber

$8 \mathrm{SE}: \quad \quad \#>(\mathrm{a}: \mathrm{ch}$ qua/) \#

9 FU: nich was de (wie/) > ja: zum bei"spie: 1-*

$10 \mathrm{BA}$ : alleinsein un so-

11 OT:

$12 \mathrm{NW}:$ $\mathrm{K}:$

$\geq$ (onatsch)

$>$ meinst $=e$

$>$ ha des weiß man ia

13 SE:

14 BA: nicht

15 OT: manchmal se"lber nich-

des glaub ich (...) nicht

ja genau * ja Gudrun STösST ATEM AUS

Frau Funke argumentiert weiter, viele medizinische Untersuchungen hätten kein Ergebnis gebracht, und sie rate Frau Senft zu einer psychosomatischen Klinik. Diese glaubt aber nicht, daß sie große kümmernisse habe; es sei der kleinscheiß, der einen ärgert, aber nich so, daß er mich psychisch belastet. Frau Funke kehrt auch dieses Argument für ihre These um: $d u$, wenn de des wüßtest, dann brauchtest du ja kein $N N$ (= Krankheit) zu kriegen. Der Disput geht so aus, daß Frau Senft Frau Funke bittet, vierzehn Tage bei ihr übernachten zu dürfen, um abzuklären, ob nicht doch eine materielle Ursache dahintersteckt. Frau Funke geht auf diesen Vorschlag ein.

Wesentlich für die Literaturgruppe scheint uns, daß Diskrepanzen in der Bereitwilligkeit bzw. Fähigkeit, über eigene Probleme zu sprechen, indirekt und argumentativ behandelt werden. Im vorliegenden Fall läßt man das eigentliche Konfliktthema (das Tabuhalten persönlicher Probleme) auf sich beruhen und zeigt sich kooperativ-helfend bei der praktischen Lösung des persönlichen Problems. Die reflexive und kooperative Art des Umgangs miteinander kommt auch im Diskussionsstil zum Ausdruck: Die Frauen hören einander zu, sie verwenden die Argumente der Gegnerin für eigene Argumentationsziele und sie sprechen oft metakommunikativ über argumentative Tätigkeiten und den argumentativen Status von Behauptungen. 


\subsection{MEINUNGSUNTERSCHIEDE UND DIVERGENZEN DER WERTUNGSSYSTEME}

Manche Konflikte entstehen aufgrund unterschiedlicher Wertungssysteme, die mit generellen Lebensorientierungen oder Lebenssituationen der Frauen zusammenhängen.

\subsubsection{BASTELGRUPPE: AUFDECKUNG DER DIVERGENZEN DURCH STREIT}

Konflikte der genannten Art treten in der Bastelgruppe häufig auf und führen oft zu expandierten Normendebatten, meist in Verbindung mit Tratsch und/oder Berichten/Erzählungen aus Filmen und Illustrierten. Sie sind immer verbunden mit Bewertungen, mit sozialer Typisierung bzw. Kategorisierung und mit mehr oder minder expliziten Abgrenzungen von negativ bewerteten Rollen- und/oder Statuskategorien bzw. mit kategoriengebundenen Handlungsweisen. 10

Die in der Gruppe übliche Ordnung nach Statuskategorien ist dreigliedrig; in Relation zu 'uns' gibt es Statuskategorien 'oberhalb' und 'unterhalb von uns'. Eine besondere Bedeutung kommt in der Gruppe einer Statuskategorie zwischen 'uns' und 'oben' zu, den möschte-sein bzw. foine leut. Damit werden Leute bezeichnet, die aus 'unserer Mitte' stammen, sich nach 'oben' orientieren und sich von 'uns' distanzieren, denen jedoch für den sozialen Aufstieg die ideellen und materiellen Voraussetzungen fehlen. Sie werden immer negativ bewertet, ihre Handlungsweisen als vorgebliche entlarvt.

Das folgende Beispiel stellt einen Konfliktfall dar, bei dem es um die implizite $\mathrm{Zu}$ ordnung zur Kategorie der foine leut geht. Den Konflikt verschärfend kommt hinzu, daß das betreffende Gruppenmitglied sein Aufwärtsstreben vor den anderen zu verbergen sucht, alle aber davon wissen. Der Konflikt um divergierende soziale Orientierungen wird also überlagert durch einen Konflikt wegen der Verletzung von Gruppenregeln (vgl. oben 2.2.1. Tabuhalten von Problemen). Die Bearbeitungsform ist der offene Streit. Offenem Streit gehen meist andere Formen der Konfliktbearbeitung voraus, wie Frotzeln (vgl. oben 2.2.) oder Tratsch (vgl. unten 2.4.); Streit bricht nach länger schwelendem Konflikt bei vergleichsweise nichtigen Anlässen aus und kann zu kurzen oder ausgedehnten heftigen Wortwechseln führen, meist verbunden mit derben Beschimpfungen. So auch im folgenden Beispiel.

Frau Wichmer (WH) zeigt den anderen Frauen Photos von ihren fünf Katzen. Da die Photos in ihrer Wohnung gemacht wurden, gewährt sie den Frauen unbeabsichtigt Einblick in ihren Privatbereich. Auf einem der Bilder ist viel von Frau Wichmers teuer eingerichteter Wohnung zu sehen, eine Katze räkelt sich auf einem rotplüschigen Sofa, und Frau Wichmer sitzt in einem langen, dunkelrot glänzenden Kleid daneben. Die Bilder werden von einigen Frauen überschwenglich kommentiert; nur Frau Kunz (KU) macht bissige Bemerkungen. Als sie das letzte Bild betrachtet, beginnt folgender Abtausch:

10 Zu Rollen- und Statuskategorien vgl. W. Kallmeyer (1988). Zum Begriff kategoriengebundener Handlungsweisen vgl. W. Kallmeyer/I. Keim (1989b). 
$1 \mathrm{KU}:$ <i"nken mer kennt määne des wär die Nitribit IACHT BOSHAFT

2 IN: ja

3 WH: \# oh menschenskind her* < a"rschloch geb doch ruh \# * K: \# BÖSE GIFTIG \#

$4 \mathrm{WH}: \quad$ is des e bledi kuh hert

$5 \mathrm{KU}: \#>$ her isch kumm glei $(\ldots) \#$

$\mathrm{K}:$ \# DROHEND \#

$6 \mathrm{KU}$ : WENDET SICH AN ANDERE FRAU UND KOMMENTIERT WEITER DIE BILDER

Das Referenzobjekt in Frau Kunzes erstem Turn ist vordergründig Frau Wichmers Katze. Doch auf dem Hintergrund der in spielerischen Andeutungen üblichen Verwendung von weiblichen bzw. männlichen Tieren als Metaphern für Frauen oder Männer bleibt die Referenz in gewisser Weise ambivalent. Der vorsichtig durch Irrealis formulierten $\mathrm{Zu}$ schreibung liegt eine implizite Vergleichsrelation zugrunde: Die Katze (oder Frau Wichmer?) räkelt sich so, daß man meinen könnte, sie wäre die Nitribit. Das Vergleichsobjekt ist eindeutig negativ. Der Name referiert auf ein in sogenannten prominenten Kreisen verkehrendes Callgirl aus den 60er Jahren, das in Frankfurt ermordet wurde und dessen Mord nie aufgeklärt wurde. Der Fall wurde durch aufsehenerregende Presseberichte bundesweit bekannt. Die Referenz auf dieses Callgirl in Zusammenhang mit Frau Wichmers teurer Wohnungsausstattung schafft einen neuen Deutungsrahmen für die außerhalb der sozialen Normalität stehende Wohlhabenheit von Frau Wichmer und suggeriert als Erklärung dafür: Wohlhabenheit durch Prostitution und zwar Prostitution in höheren Kreisen. In ernster Modalität bedeutete dies eine tiefgehende Beleidigung von Frau Wichmer. Doch Frau Kunz hält durch das, wenn auch boshafte Lachen, die Modalität offen und schwächt die implizit bleibende Beleidigung durch vorsichtiges Formulieren ab (Irrealis, Vergleich). Frau Kunzes Bosheit kann hier nicht auf dunkle Punkte in Frau Wichmers Wohlhabenheit zielen (denn alle wissen, daß sie durch langjähriges Führen eines gut gehenden Lokals und nicht durch Prostitution zu ihrem relativen Wohlstand kam), sondern der zweite Aspekt der Nitribit-Metapher, die -Orientierung auf ,höhere Kreise' hin ist der eigentliche Kritikpunkt; diese Orientierung wird auf dem Photo durch Frau Wichmers aufwendige Wohnungseinrichtung mit teuren Gegenständen, Teppichen und Antiquitäten offenbart. Frau Kunze kritisiert auf indirekte Weise, daß Frau Wichmer durch das Sichumgeben mit solchen, nicht zu ihrer Welt gehörenden Statussymbolen mehr scheinen will, als sie ist, sich geschmacklich von ihrer Welt distanziert. Auf Frau Kunzes Angriff zeigt sich Frau Wichmer stark getroffen; sie reagiert wütend mit einem harten Schimpfwort (arschloch) und der Aufforderung an Frau Kunz, Anspielungen und Unterstellungen der gemachten Art zu unterlassen (Z. 3). Auf Frau Wichmers Beschimpfung reagiert Frau Kunz jetzt ebenfalls wütend mit der Drohformel her isch kumm glei (...), die sie vermutlich durch niwwer komplettierte. Es handelt sich hier um eine den Mitgliedern geläufige Drohformel, die die Androhung von Schlägen beinhaltet, die man auszuführen ankündigt, wenn der Kontrahent nicht zurücksteckt. 
Frau Wichmers Replik auf die Drohformel stellt eine deutlich schwächere Form der Beleidigung dar, d.h. sie hat die implizite Kritik erfaßt und steckt zurück. Auf einer Skala von Schimpfwörtern für Frauen gehört bledi kuh zu den milderen Formen, am anderen Ende der Skala liegen die eher harten Schimpfwörter mit Bezeichnungen für Prostituierte (z.B. hur). nitribit in Frau Kunzes erstem Turn gehört nicht zu den Schimpfwörtern, bezeichnet jedoch eine soziale Kategorie, auf die sich in der Schimpfwortskala die besonders harten Schimpfwörter beziehen. D.h. Frau Kunz beginnt die Auseinandersetzung auf der Kategorisierungsebene mit einer stark negativ bewerteten sozialen Kategorie. Mit dieser wissentlich falschen, allerdings durch die halbspielerische Modalität abgeschwächte Negativkategorisierung straft sie Frau Wichmer für ihre hier unwillentlich offenbarte, bisher verborgen gehaltene Aufwärtsorientierung.

Nach diesem Abtausch reden die beiden Frauen ca. eine halbe Stunde nicht mehr miteinander; dann wendet sich Frau Wichmer mit einer teils ärgerlich, teils scherzhaft geäußerten Drohformel nochmals an Frau Kunz und leitet damit die Beilegung des Konflikts ein: isch schlach der noch doi brill runner her wenn $=d$ so $=e$ gro $\beta i$ gosch hosch. Darauf reagiert Frau Kunz nonverbal, sie schneidet Fratzen. Anschließend lachen beide Frauen; der Streit ist damit beendet.

In der Beteiligungsweise am Streit erweist sich Frau Wichmer als echtes Gruppenmitglied (Kenntnis der Drohformel, Kenntnis der Regeln für Deeskalierung des Konflikts, Verstehen der Nitribit-Metapher und der damit implizierten Kritik) und stellt damit selbst einen offensichtlichen Gegensatz her zu dem an ihr kritisierten Aufwärtsstreben. Sie akzeptiert die normative Basis der Kritik und leitet später die Beilegung des Konflikts ein.

Dem Streit liegen zwei sich überlagernde Konflikttypen zugrunde, das erklärt seine Heftigkeit. Auffallend an der Durchführung des Streits sind folgende Merkmale:

- Ärger und Aggression werden von beiden Konfliktparteien offen geäußert durch Beschimpfungen und Drohformeln. Dies ist auch bei anderen Streitereien der Fall.

- Das Kritikobjekt (Aufwärtsorientierung) und die damit verbundene negative soziale Kategorisierung wird mehrfach verschlüsselt (Vergleich, mehrdimensionale Metapher,Irrealis) eingeführt. Die Indirektheit der Kritik hat mit der Tabuisierung des Themas durch Frau Wichmer zu tun.

- Die Strafe für die Tabuisierung erfolgt durch bewußte, allerdings spielerisch gemilderte Falschkategorisierung (Prostituierte) von Frau Wichmer.

Die Absage von der inkriminierten Haltung erfolgt implizit durch das Praktizieren gruppenspezifischer Streitverfahren.

\subsubsection{LITERATURGRUPPE: DAS VERDECKTHALTEN VON DIVERGENZEN}

Ähnliche Konfliktanlässe, die in einer sozialen Absetzung gegenüber den Gruppenmitgliedern gründen könnten, werden anders behandelt. Nur gruppenextern wird eine Frau, die sich für etwas Besseres hält als die Nachbarschaftsumgebung ihres Stadtteils, durch eine gezierte, überfeine Sprechweise karikiert. Die Frauen haben - wie gesagt - unterschiedliche Ansichten über das Frauenbild, die Eherolle, Erziehungsprinzipien, literari- 
sche Vorlieben usw. Es bestehen auch unterschiedliche finanzielle Ressourcen der Familien und damit ein unterschiedlich aufwendiger Lebensstil, der von den Frauen aufmerksam registriert wird (s.u.).

Wo unterschiedliche Ansichten und Einstellungen zur Lebenspraxis in den Gesprächen thematisch auftauchen, werden sie auf eine Weise behandelt, die die Differenzen markiert, jedoch den Willen zeigt, darüber nicht in einen Streit zu geraten, sondern über partielle gemeinsame Ansichten zu einem Konsens zu gelangen. Durchführungsmittel sind dafür: argumentativer Diskurs, die Zurücknahme einer negativen Wertung, das Ausnützen sprachlicher Abschwächungsformen bei Kritik, Andeutungen der Meinungsdivergenzen in frotzelnder, aber nicht-aggressiver Form; in einem Falle die Transposition einer Meinungsdifferenz in einen bildlich-metaphorischen Bereich mit komischer Wirkung, d.h. in anderer Modalisierung.

Zunächst soll ein Beispiel diskutiert werden, das auf unterschiedlichen Vorstellungen der Frauen über den Aufwand bei Festen beruht. Mehrere Frauen reagieren kritisch auf die Mitteilung von Frau Sternberg und Frau Kerk, daß sie sehr viele Gäste bei Hochzeitsfeiern eingeladen haben:

1 ST: und für hu"ndert personen * äh hatten se ja vo"rjehabt * langt des ja

2 ST: nich hinten un nich vornet

3 FU: \#بhu:ndert personent\# ach größer geht=s nich

K: \# ERSTAUNT \#

4 ST: neet

$5 \mathrm{KE}$ ah ja na: was willst du- wir warn hundertzwa:nzich $\downarrow$

6 BA: $\quad$ ija inr seid au"ch *

7 BA: bekloppt >hi hi entschu"ldijel



9 GR: (......) find ich au"ch was soll denn das eigentlich $\downarrow$

$10 \mathrm{BA}:$ ja das find ich auch

11 FU: $(\ldots \ldots)$ hochzeitsreise für zehntausend mark finanziert $\downarrow$ also

12 FU: ehrlich Erika * w/ warum muß denn so" ein rie:senpolterabend seint

Nicht nur Frau Bareis hält die Zahl von über hundert Personen für zu groß, sondern auch Frau Funke und Frau Gries. Aber diese realisieren ihre Kritik zunächst in ironischer Form (ach größer geht $=s$ nich, Z. 3 ) bzw. in mehreren Anläufen zunehmender Mißbilligung. Frau Gries kleidet ihre kritische Stellungnahme in eine beziehungssichernde Entschuldigungsformel und in zwei Sätze, mit denen sie ihre eigene Haltung mittels eines Verbs des Sagens oder Denkens thematisiert (auch ich muß sagen, find ich auch, Z. 8f. ). Erst dann gibt sie eine Stellungnahme in Form einer rhetorischen Frage ab: was soll denn das eigentlich? (Z.9). Die spontane und relativ starke Bewertung mit dem Adjektiv bekloppt (Z. 7) wird von Frau Bareis erstens durch ein kurzes Lachen abgeschwächt, dann aber explizit durch eine Entschuldigung zurückgenommen. 
Die Angegriffene verteidigt sich, indem sie die Argumentation ihrer Kinder weitergibt, mit der sie sich identifiziert (und ich sag aber gleich vorneweg, daß se mir ja letzten endes ingendwo eingeleuchtet hat). Dabei bekommt sie Unterstützung von Frau Sternberg, die ja ebenfalls ein Fest mit sehr vielen Gästen ausgerichtet hat. Die Gegenfront verzichtet auf ihren Widerspruch, außer einer kurzen, formelhaften und in Flüstern übergehenden Wertung (d.h. mit zurückgenommener Adressierung) von Frau Funke:

$1 \mathrm{KE}$ : tund im nu: waren wir/ $\rightarrow$ und wir ham=s also mal durchgezählt *

2 KE: hu"ndertse"chsundzwanzig leute $\downarrow$

3 FU:

haha $\rightarrow$ des is ja * \#wahnsinn\#

K:

\# GEFLÜSTERT \#

Hinter den nicht ausgetragenen Meinungsunterschieden über die Angemessenheit der Gästezahl steht wahrscheinlich die Befürchtung, einzelne Mitglieder könnten sich an anderen sozialen Welten orientieren.

Die Frauen bekennen sich einerseits zu ihren unterschiedlichen Auffassungen, sie diskutieren sie aber nicht aus. Zugrundeliegende mögliche Gründe für das unterschiedliche Verhalten (unterschiedliche soziale Verpflichtungen durch die Berufe der Männer) bleiben dadurch verdeckt. Diese Themen werden in ihrer Relevanz herabgestuft, um den Zusammenhalt der Gruppe für die ihr wichtigen Themen zu gewährleisten.

In einem anderen Fall, bei dem auch das Verfügen über finanzielle Mittel im $\mathrm{Zu}$ sammenhang mit unterschiedlichen Erziehungsmaximen ("verwöhnen" oder "knapp halten") steht, wirft Frau Ott der Familie Kerk vor, sie habe ihren Kindern zum 18. Geburtstag ein Auto geschenkt, während sie und ihr Mann sich das nicht leisten könnten und es auch nicht machen würden, selbst wenn wir kö"nnten:

1 OT: da sacht \# sie"\# dann oder ihr ma"nn * wir ham des ganz ei"nfach

2 or: geklärt *

$\mathrm{K}: \quad$ \# $\quad$ FRAU KERK \#

3 OT: wir ham denen zum achzehnten oder zum neunzehnten lebensjahr $n$

4 OT: au:to geschenkt $\uparrow$ und dann mußten/ oder $\mathrm{n}$ auto ham se gekriggt $\uparrow$

5 oT: und dann mußten sie * eben das entsprechend se"lber versorgent

6 OT: net tund da

7 or: geht=s dann schon lo:s $\uparrow$ (es is vielleicht/)

$8 \mathrm{KE} \mathrm{ja}$ also qa"nz so stimmit=s ni"cht * das

9 OT: ja

$10 \mathrm{KE}:$ is das is/ eh da"s was ich sa:chtet * das ham wer erst im laufe

$11 \mathrm{KE}$ : der zei:t gemerkt $\downarrow$-erstens hat kei"ns von den kindern des auto

$12 \mathrm{KE}$ : gesche"nkt bekomment sonderm sie mußten also zwei drittel selber

$13 \mathrm{KE}$ : aufbringen $\downarrow$ * und des mußten sie sich müh:sam verdie:nen * äh

$14 \mathrm{KE}$ : und leider ham wer bei der U"lla des ni"cht gemacht [...] 
Frau Ott schwächt von vornherein ihren Vorwurf durch Selbstkorrekturen ab: oder ihr ma"nn (die Adressatin wird aus der Kritik herausgenommen); $n$ auto geschenkt [...] oder $n$ auto ham se gekriggt (Abschwächung des Vorwurfs des Verwöhnens). Frau Kerk weist den Vorwurf zurück, indem sie mehrere Umstände anführt, die ihre Handlungsweise nicht als Verwöhnen erscheinen lassen; im Gegenteil, sie bezichtigt sich im weiteren Gespräch, bei ihrer Tochter zu streng verfahren zu sein, so daß dies ihren Lebensweg negativ beeinflußt habe. Wie im vorigen Beispiel geht das Gespräch zu einem neuen Subthema über.

An diesem Beispiel ist - außer den für die Gruppe charakteristischen Abschwächungsformen bei kritischen Beziehungsakten - zu erkennen, daß ein möglicher zugrundeliegender Normkonflikt (verwöhnen vs. knapp halten) abgestritten wird, weil er einen wesentlichen Aspekt des Selbstbildnisses der Gruppe betrifft. Die Angegriffene ist bemüht, nicht einer sozialen Kategorie zugeordnet zu werden, deren Angehörige ein angeberisches, den Reichtum zur Schau stellendes soziales Verhalten vorgeworfen werden kann.

Im Unterschied zur Bastelgruppe spielen für diese Frauen soziale Statuskategorien (sich absetzen gegen 'oben' oder 'unten') keine besondere Rolle, sie klingen nur verhalten an in Gesprächspassagen wie oben.

Ein brisanteres Thema ist in der Literaturgruppe die Frauenrolle, d.h. das Maß an Selbstbestimmung, das eine Frau ihrem Mann gegenüber fordert. Dieses Thema wurde für zwei Beteiligte in sehr schwierigen Lebenslagen besprochen und von der ganzen Gruppe im Sinne der Betroffenen gelöst (die Frauen sprechen nicht gerne darüber). Unterschiedliche Sehweisen in Bezug auf die Frauen- und Partnerrolle werden scherzhaft, frotzelnd besprochen. Als Frau Kerk z.B. einsieht, daß sie sehr viel öfter als ihr Mann abends alleine zu Hause ist, wird sie aufgezogen; aber auch diejenige, die sie aufzieht, wird scherzhaft darauf hingewiesen, daß es bei ihr noch nicht so lange her ist, daß sie sich in einer Sache gegen ihren Mann durchsetzte:

1 BE: das wi"rd noch bei der Erika > des dauert noch

2 OT:

\#ku=mal

$\mathrm{K}:$

\# LACHEND \#

3 OT: die Hilde die spricht jetzt au"ch schon ganz anders\#

Umgekehrt frotzeln die Frauen, die eine eher traditionelle Auffassung von der Frauenrolle haben, wenn sie z.B. hören, daß eine Teilnehmerin ein Frauenseminar besuchte: des müßt ihr mühsam lernen?

Auch bei Themen, die keine grundsätzlichen sozialen Orientierungen des Lebensstils oder des inneren Kerns des Selbstverständnisses betreffen, bemühen sich die Frauen, auftauchende Meinungsverschiedenheiten in einem Konsens oder in einer Beziehungsharmonie aufzuheben. Manchmal entschlüpft einer Frau der Literaturgruppe eine kritische Bemerkung, die auf anderen Wertungskriterien beruht und von der sich die Sprecherin nachträglich distanziert, weil sie merkt, daß sie zu weit gegangen ist. Es beginnen dann Reparaturen der Imageverletzung, so wie im vorletzten Beispiel Frau Bareis sich für den Aus- 
druck bekloppt entschuldigt hatte. Eine besonders aufwendige Wiederherstellung einer guten Beziehung kann man im folgenden Beispiel sehen, bei dem Frau Ott eine kritische Bemerkung unterlaufen war:

1 BA: hab ich euch erzählt daß ich mein funkkolleg bestanden habe $\uparrow$

$2 \mathrm{BA}$

mit ner drei:

3 OT:

on des-s schwacht

$4 \mathrm{NW}$ : bravo wo is der sekt $\uparrow$

5 FU: LACHT

6 OT: des war wieder dumm von mir aber des find ich echt super

7 or: to"ll kla"sse schön

Die zu abschätzige Beurteilung hebt Frau Ott mit einer Selbstbeschuldigung auf und versucht sie durch eine Häufung von hyperbolischen, zum Teil der Sprache der Jugendlichen (der eigenen Kinder) entlehnten Adjektiven ins Gegenteil zu ändern. Frau Ott erläutert aber nicht, warum ihr die Note "drei" als eine "schwache" Leistung erscheint. ${ }^{11}$

Für die Gruppe typisch scheint uns auch das Sprechen über Konflikte in einer bildhaften Redeweise zu sein. Das folgende Beispiel entstand aus einer Meinungsverschiedenheit zwischen Frau Funke und Frau Kerk darüber, ob man eine Erzählung von Handke vorlesen sollte oder nicht. Frau Funke war dafür, Frau Kerk dagegen. (Beide schwächten aber schon bei der Diskussion über die Lektüre ihre Vorschläge bzw. Einreden ab.)

Die Vorlesende ( = Frau Funke) sitzt neben der Opponentin (Frau Kerk). Sie unterbricht das Vorlesen, ohne daß jemand unmittelbar zuvor etwas gesagt hat, sie lacht kurz und sagt dann:

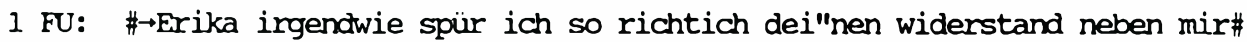
$\mathrm{K}:$ \# LACHEND \#

2 FU:

$3 \mathrm{KE}$ : ha ha ha ha ha

(du bist?das ist) so richtich witzich hahaha ha

11 Wenn die Frauen der Literaturgruppe sachlich widersprechende oder verhaltenskritische Äußerungen machen, dann nehmen sie ihnen die aggressive Schärfe und schwächen einen möglichen Imageangriff ab. Sprachliche und kommunikative Mittel dafür sind:

- Redeeinleitungen mit $d u$ und Adressierungen (z.B. kuck mal);

- Kosenamen: schätzchen;

- lexikalische Abschwächungen: vielleicht, wahrscheinlich, mal;

- Ausdrücke der propositionalen Einstellung: ich meine, ich denke, ich weiß nich;

- mehrfach abschwächende metakommunikative Äußerungen: also ich würde da aber doch auch erheblich einspruch erheben;

- die eigene Meinung als Fragesatz formulieren: war des nicht auf karten geklebt? (gegen die Meinung: auf taschentücher gedruckt);

- inhaltlich abschwächende Selbstkorrekturen;

- Konsensfeststellungen in nebensächlichen Aspekten eines Sachverhalts;

- Themenwechsel zu einem unverfänglichen Thema. 




15 GR: da hinsetzen-

$16 \mathrm{SE}:$ spürbar-

17 OT:

18 FU:

LACHT ja wem=s gefällt

K:

\#es is ja auch kei"ne \# LACHEND \#

19 FU: kriti"k verstehst du-\#

Die mögliche konfliktäre Beziehung wird von Frau Funke von vornherein durch ein lachendes Sprechen abgeschwächt. Frau Kerk geht auf diese freundliche Art der Thematisierung des Konfliktpunktes ebenfalls durch ein Lachen ein. Die anderen Teilnehmerinnen greifen nun das Ungewöhnliche an der Wahrnehmung der ablehnenden Haltung von Frau Funke auf; sie bringen die kritische Beziehung in ein Bild (strö:mt förmlich) und machen Vorschläge, wie die räumliche Nähe und damit das Strömen des Widerwillens aufgehoben werden können (wahrscheinlich bewegt sich Frau Kerk von Frau Funke weg, dazu paßt: bleib doch hier, setz dich woanders hin). Nun haben zwei Teilnehmerinnen die Idee, daß das Sitzen im Rücken der Kontrahentin noch schlimmer ist (weil die Vorleserin sie nicht sehen kann), und sie formulieren ihren Einfall fast gleichzeitig mit fast denselben Worten. Das löst eine weitere lange Lachsequenz aller Beteiligten aus. Die Vorstellung eines psychisch-physischen Flusses von einer Person zur anderen und die Übernahme dieser Vorstellung bei veränderten situativen (räumlichen) Bedingungen machen den thematischen Kern der Scherzinteraktion aus, in der der Streitpunkt untergeht. Dennoch bestätigt die Initiatorin des Zwischenfalls zum Schluß metakommunikativ, daß ihre Bemerkung keine Kritik gewesen sei.

Insgesamt achten die Frauen sehr darauf, daß sie sich mit negativen Gefühlen und Vorwürfen nicht zu nahe treten. Offen aggressive Akte, wie sich anschreien oder Beschimpfungen kommen nicht vor. Distanzwahrung ist die Regel nach der Lebens- und Meinungsdivergenzen, die als gravierend angesehen werden, behandelt werden. 


\subsection{POTENTIELL GRUPPENSPRENGENDES VERHALTEN}

Hier behandeln wir schwerwiegende Verstöße gegen Gruppennormen, die tendenziell zur Gruppenauflösung führen bzw. führen würden, wenn sie vorkämen. Gemeint sind Konflikte, bei denen Normen verletzt werden, die zu den konstitutiven Gruppennormen gehören. Bei Normverstößen dieser Art unterscheiden sich die untersuchten Gruppen erheblich. Verstöße, die in der Bastelgruppe zu den gruppensprengenden gehören, wie das materielle Ausbeuten anderer Mitglieder, treten in der Literaturgruppe nicht auf und Verstöße, die dort gruppengefährend sein können, z.B. das übermäßige Lamentieren, spielen in der Bastelgruppe eine wesentlich geringere Rolle. Hier gehören sie zu den Verstößen, die ähnlich wie die in Kapitel 2.2.1. dargestellten bearbeitet werden.

\subsubsection{BASTELGRUPPE: AUFDECKEN VON SCHWEREN NORMVERSTÖSSEN}

Schwerwiegende Konflikte werden meist durch hartes Frotzeln (vgl. 2.2.1.) und durch Tratsch bearbeitet. $\mathrm{Zu}$ den konstitutiven Merkmalen von Tratsch gehört, daß die Betratschte ( $=$ Tratschobjekt) abwesend ist, daß sie den am Tratsch Beteiligten (= Akteuren) bekannt ist und eine/mehrere Verhaltensweisen des Tratschobjekts meist anhand von Ereignissen dargestellt und negativ bewertet werden.

Beim Einsatz von Tratsch zur Behandlung von Konflikten sind zwei Typen zu unterscheiden in Bezug auf die Konstellation der Beteiligten und auf das Tratschobjekt:

a) Tratsch über ein Gruppenmitglied (Tratschobjekt) und sein Vergehen unter Beteiligung eines Mitglieds, von dem man annimmt, daß es dem Tratschobjekt von dem Tratsch und der Kritik an seinem Verhalten berichtet.

b) Tratsch über ein gruppenexternes Tratschobjekt mit dem Ziel, ein anwesendes bzw. beteiligtes Mitglied zu treffen, an dem dieselben Eigenschaften/Handlungsweisen kritisiert werden wie an dem Tratschobjekt.

Beides sind häufig praktizierte Bearbeitungsformen für schwerwiegende Konflikte, die aus Verstößen gegen Gruppennormen oder gegen allgemeine Normen resultieren, die sozialen Kategorien zugrunde liegen und die entscheidend für das Selbstverständnis der Beteiligten sind. Es sind Verstöße, die zu Überlegungen veranlassen, ob die betreffende Person noch 'zu uns gehört'.

Interessant ist vor allem der unter b) angeführte Tratsch, da hier von der/n Kritikerin/nen und der Zielperson (der Beteiligten, der der Tratsch gilt) wesentlich höhere kommunikative und interpretative Leistungen erbracht werden müssen, um das Tratschziel zu erkennen, als beim Tratsch unter a), in dem viel offener, direkter und schonungsloser geurteilt werden kann. Die Kritisierten zeigen in Tratschsituationen vom Typ b) vor allem folgende Reaktionen: sich dumm stellen, d.h. die Kritisierte gibt vor, die indirekte Kritik nicht verstanden zu haben, sie beteiligt sich nicht mehr am Tratsch oder nur in den harmloseren Tratschpassagen; zum anderen das Antreten der Flucht nach vorn, d.h. die 
Kritisierte kooperiert mit den Kritikerinnen durch Übernahme von deren Perspektive und Bewertung auf die kritisierte Handlungsweise und distanziert sich so indirekt von ihrer eigenen Handlung, auf die die Kritik ja zielte. Dabei können Perspektivenübernahme und Distanzierung von der eigenen Handlungsweise nur vordergründig sein im Sinne einer "'working' acceptance" (dt.: "Arbeitskonsens", Goffman 1971:17) und nicht wirkliche Einsichten bzw. Absichtserklärungen zu Verhaltensänderungen implizieren.

Soll die indirekte Kritik ankommen, d.h. verstanden werden, und will die Kritisierte die verdeckte Bearbeitung des Problems, dann haben Kritikerin und Kritisierte komplexe interaktive Aufgaben zu bewältigen. Die Kritikerin muß vor allem

- das Tratschthema der Zielperson des Tratschens unauffällig anbieten,

- deren Kooperation sichern,

- in unauffälliger Weise die kritisierte Handlungsweise fokussieren und ein "intendiertes Mehrfachverständnis" (Schlieben-Lange, 1983:92) signalisieren,

- die Kritisierte behutsam zur Beurteilung führen und

- die Übereinstimmung in der Beurteilung sichern.

Die Aufgaben der Kritisierten bestehen vor allem in

- dem Erkennen, da $\beta$ es bei der Bearbeitung des Falles zwei Ebenen gibt,

- dem Erkennen der Analogie zwischen der inkriminierten Handlungsweise des behandelten Falles und der eigenen Handlungsweisen,

- der Stellungnahme zur auf der zweiten Ebene gegebenen Kritik.

Zu dieser Form der Konfliktbearbeitung folgt nun die kurze Analyse eines Textausschnittes, der aus Raumgründen nur an den entscheidenden Stellen eingehender untersucht werden kann. Die Hauptakteurinnen sind Frau Zimmermann als vorrangige Tratschproduzentin und Frau Kunz als vorrangige Rezipientin. Vor diesem Treffen war Frau Kunz bereits mehrmals Tratschobjekt in mehr oder weniger ausgedehnten Tratschpassagen, in denen es vor allem um Frau Kunzes gruppenschädigende Handlungsweise geht, die als $a b$ sahnen bezeichnet wird. absahnen bedeutet das rücksichtslose Nutznießen von Leistungen und Anstrengungen anderer. ${ }^{12}$ Tratschobjekt im vorliegenden Ausschnitt ist die gruppenexterne Frau Xaver. Zielperson des Tratsches ist die am Tratsch beteiligte Frau Kunz.

\section{a) Herstellung der Tratschgelegenheit}

Vor dem Tratsch über Frau Xaver ist die Interaktionsform Tratsch bereits etabliert. Das Tratschobjekt Frau Xaver wird von Frau Kunz in einem anderen thematischen Zusammenhang selbst eingeführt. Schwierigkeiten bei der namentlichen Identifizierung der Frau

12 So nahm Frau Kunz bereits mehrmals an Gruppenfeiern teil, versorgte sich überreichlich mit von der Gruppe aufgebrachten Speise- und Getränkeangeboten, ohne selbst etwas zum Gruppenfest beigetragen zu haben. 
bieten Frau Zimmermann Anlaß zu einer Neufokussierung: Frau Xavers Geiz veranlaßte sie, ihr einen Spitznamen zu geben. Mit der Thematisierung des Geizes bewegt sich der Tratsch bereits sehr nahe an dem späteren Tratschthema absahnen.

\section{b) Sicherung der Kooperation der Zielperson}

An Frau Kunz adressiert, bietet Frau Zimmermann an, das Ereignis zu erzählen, das sie zur Vergabe des Spitznamens veranlaßte. Obwohl Frau Kunz dieses Angebot nicht ratifiziert, startet Frau Zimmermann ihre Erzählung und es gelingt ihr, das Interesse bei Frau Kunz zu wecken und ihre Kooperationsbereitschaft zu sichern, die sie durch die offene mißbilligende Beurteilung des Verhaltens von Frau Xaver kundtut.

\section{c) Fokussierung der kritisierten Handlungsweise'absahnen' und Signalisienung einer zweiten Verstehensweise}

Bereits gegen Ende von Frau Kunzes Beurteilung liefert Frau Zimmermann ohne Ankündigung und nur durch einen Aufmerksamkeitsappell markiert, weitere Informationen zum Tratschobjekt.

1 2I: is klar/ $<$ hert emol

$2 \mathrm{KU}:$ \#<diess gschdört wenn se wege zäh penning kilometerweit rennt\# $\mathrm{K}$ : \# VEHEMENT \#

$3 \mathrm{ZI}$ : $\rightarrow$ mer mer ware doch neilisch $\mathrm{mi}=\mathrm{m}$ freizeithaus + in de: *

$4 \mathrm{KU}: \mathrm{a}^{\prime c h}$ gott $\downarrow$

$5 \mathrm{ZI}$ : milschzentrale $\uparrow$ wer is kumme $\uparrow$ \# die (SPITZNAME) $\downarrow$

$6 \mathrm{KU}:$

$7 \mathrm{IN}:$ K: aha ja \#nää:\#

die war au

\# ERSTAUNT, EMPÖRT\#

$8 \mathrm{ZI}: \quad$-die (SPITZNAME) $\rightarrow$ is kumme schbäder

$9 \mathrm{kU}: \quad \leq \mathrm{ia}$ " die" /

10 IN: debei $\uparrow$ is die sunscht net im

11 2I:

12 IN: freizeithaus $\uparrow$

$13 \mathrm{KR}$ : we"r is die (SPITZNAME) <die" geht gar net nau"s

Durch größere Lautstärke, Adressierung der Mitteilung an alle (im Gegensatz zur Adressierung vorher an Frau Kunz), höheres Sprechtempo, größeres Engagement in der Stimme, zeigt Frau Zimmermann an, daß sie etwas Bedeutsames mitzuteilen hat und sichert sich so die Aufmerksamkeit der anderen.

Die Mitteilung in Form eines Geschichtenkondensats hat eine dreiteilige Struktur: einen narrativen Satz, der auf Ort, Personen und Umstände des Geschehens referiert; eine Frage, die den Bezug zwischen dem bisherigen Tratschobjekt und der jetzigen Szenerie herstellt und die inkriminierte Handlungsweise verschlüsselt anzeigt; und die Nennung des 
Tratschobjekts als Antwort auf die Frage. Dieses Geschichtenkondensat ist äußerst komplex und voraussetzungsreich. Das freizeithaus ist eine kommunale Einrichtung und Frau Zimmermann ist dort regelmäßige Besucherin. Diese Einrichtung macht in Abständen Firmenbesuche des freien Speise- und Getränkeangebots wegen. Freizeitgruppen, die solche Ausflüge machen, wachen eifersüchtig darüber, daß außergewöhnliche Unternehmungen auch nur von denen wahrgenommen werden, die auch an den normalen Gruppenaktivitäten teilnehmen, nicht nur 'die Rosinen aus dem Teig picken' wollen und die harte Alltagsarbeit der Gruppenaufrechterhaltung den anderen überlassen. Solche Personen werden als "Absahner/innen" bezeichnet. Auf die Zuordnung von Frau Xaver zu dieser sozialen Kategorie zielt implizit Frau Zimmermanns Geschichtenkondensat: Frau Xaver gehört nicht zur Gruppe des Freizeithauses und nahm an einem Ausflug zur Milchzentrale mit üppigem Angebot teil.

Auffallend an Frau Zimmermanns Formulierung ist die Implizitheit der Darstellung der charakteristischen Handlungsweise, die auf die intendierte soziale Kategorie verweist und die ausbleibende Bewertung dieser Handlungsweise. Für Tratschmitteilungen und Tratscherzählungen allgemein ist üblich, daß bereits am Anfang wertende verbale Hinweise auf das moralische Urteil gegeben werden, auf das der Klatschproduzent seine Darstellung begründet. ${ }^{13}$

Auch auf der prosodischen Ebene schwingt keine Empörung oder Abwertung mit, es wird nur der Eindruck von Bedeutsamkeit und Nachdrücklichkeit erweckt. Die hohe Implizitheit und die unübliche Zurückhaltung bei der Bewertung können als erste Signale verstanden werden, da $\beta$ es sich hier nicht um einen normalen Tratsch handelt, sondern da $ß$ zusätzliche Bedeutung übermittelt werden soll, daß ein "Mehrfachverständnis" intendiert ist. Der Zusammenhang zwischen beiden Verstehensebenen ist die Analogie von dargestellter Handlungsweise und sozialer Kategorie, zu suchen ist die gemeinte Person auf der zweiten Ebene.

In ihrer Reaktion auf Frau Zimmermanns Geschichtenkondensat zeigt Frau Kunz durch Akzentuierung, Überdehnung und auslaufendes Verhauchen der Negationspartikel nää Erstaunen und Empörung und gibt damit zu verstehen, daß sie die Implikationen in Frau Zimmermanns Kondensat, das 'Skandalon', soweit es sich auf Frau Xaver bezieht, verstanden hat. $\mathrm{Ob}$ sie die Hinweise auf eine zweite Verstehensebene hier bereits voll realisiert, ist nicht eindeutig zu klären. Auffallend ist jedoch, daß sie als einzige der Gruppenmitglieder reagiert - läßt man Inkens Nachfrage außer Betracht - und zwar sehr schnell und expressiver als Frau Zimmermann. Mit ihrer folgenden nachdrücklich und lauter geäußerten, allerdings abgebrochenen Äußerung ja" die/ hat sie möglicherweise eine Charakterisierung von Frau Xaver intendiert. Der frühe Abbruch kann durch die parallele Äußerung von Frau Zimmermann, einer nachdrücklichen Bestätigung des Skandalons, bedingt sein; es kann aber auch sein, daß Frau Kunz mit leichter Verzögerung die Hinweise auf eine zweite Verstehensebene realisiert hat, die Analogie zwischen dem dargestellten Fall und sich selbst gesehen hat, und daß sie daraufhin überrascht und betroffen schweigt. Wie der weitere Gesprächsverlauf zeigt, beteiligt sich Frau Kunz an der folgenden Identi-

13 Auch Frau Zimmermann selbst leitet andere Klatschgeschichten mit wesentlich stärkeren verbalen Indikatoren ein, die auf Bewertung und soziale Kategorisierung verweisen; vgl. auch Bergmann (1987), Kap. 5. 
fizierungssequenz nicht mehr. Erst nachdem Frau Kranz immer noch nicht verstanden hat (vgl. folgendes Transkript) und nochmals nachfragt, schaltet sich Frau Kunz erneut ein und weist Frau Kranzes beharrliches Interesse an Frau Xaver in barschem Ton (lauter und schneller gesprochen) zurück. Ein solches Verhalten ist der Gruppenbetreuerin Frau Kranz gegenüber ungewöhnlich. Frau Kunzes Zurückweisung trägt hier deutliche Züge der Überreaktion. Es scheint, als zeige sie damit, daß ihr die Behandlung des Themas unangenehm geworden ist und sie es bald beenden will.

\section{d) Beurteilung des Tratschobjekts durch die Zielperson Frau Kunz}

Im folgenden Gesprächsausschnitt nimmt die Gesprächsintensität zu. Die einzelnen Turns folgen sehr schnell aufeinander, gegen Ende überlappen sie. Die zentrale Charakterisierung von Frau Xavers inkriminierter Handlungsweise, auf die der gesamte Tratsch zielt, produzieren Frau Zimmermann und Frau Hild gemeinsam:

$1 \mathrm{KR}$ : wer is des $\uparrow$

$2 \mathrm{KU}: \quad$ Kiefer mit e $\rightarrow$ ah" die kenne sie net $t$

$3 \mathrm{ZI}$ : >hot do unne

4 2I: gewohnt <wu"=s wa"s gibt\# hot de parre

$5 \mathrm{HI}$ : \#<die" wu i"wwerall hi"ngeht-

$\mathrm{K}$ : \# BEDEUTSAM \#?

$6 \mathrm{KR}:$

$7 \mathrm{IN}:$

ah:

LACHT

$8 \mathrm{ZI}$ : wa"s >is-se aa dort $\downarrow$

$9 \mathrm{KU}$ :

$\mathrm{K}:$

nää" LACHEN

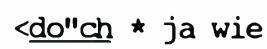

$10 \mathrm{ZI}$ : wie senioretreffe war $\uparrow$ wer is kume $\uparrow$ die (SPITZNAME) b

$11 \mathrm{HE}: \mathrm{do}: \mathrm{ch}$

$12 \mathrm{WH}$ : <wer is dest

$13 \mathrm{kU}$ : $\quad$ ou": hädd=sch gsa=s gibt widda was dolles wonn=sch do kumme

$14 \mathrm{ZI}$ : ah der=s sogar schunn vorghalte worre daß se

$15 \mathrm{KU}:$ war ou" des/s/s/s kennt=sch net hawwe

$16 \mathrm{ZI}$ : iwwer/>die die is se"hr beko"nnt daß se iwwerall hiegeht wu=s wu=s

$17 \mathrm{ZI}$ : was zu esse qibt

$18 \mathrm{kU}: \quad \leq$ nää" also des/ à ä >isch ded mer moin/

$19 \mathrm{HI}: \quad(\ldots \ldots \ldots)$

$20 \mathrm{KU}:$ swann i"sch kaffee will un e schdick kuche konn=sch mer kaafe $\downarrow$

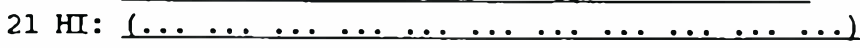


Frau Zimmermann liefert für Frau Kranz einen weiteren Identifizierungshinweis (ungefähre Angabe des Wohnorts). In dem nächsten Turn, schnell plaziert und noch mit Frau Zimmermanns Äußenung überlappend, formuliert Frau Hild, eine bisher am Tratsch nicht Beteiligte, den syntaktisch ersten Teil einer charakteristischen Handlungsweise von Frau Xaver, den Frau Zimmermann bruchlos zur syntaktisch vollständigen Form ergänzt:

$1 \mathrm{HI}:$ <die" wu iwwerall hi"ngeht

2 2I:

$<w u "=s$ wa"s gibt

Die besondere Bedeutung dieser Charakterisierung wird auf mehreren sprachlichen Ebenen angezeigt: Prosodisch bilden die beiden Äußerungsteile eine Einheit: lautere Stimme, starker Akzent auf dem ersten Wort der syntaktischen Einheit, gleiches Sprechtempo, parallele Intonationskontur (Hebung zu Beginn der syntaktischen Einheit und Auslaufen auf mittlerer Tonhöhe). Die prosodische Kontur vermittelt den Eindruck des bedeutsamen Sprechens.

Auf der Interaktionsebene bildet die gemeinsame Charakterisierung eine Art Kontrapunkt zu Frau Kunzes Versuch, das Thema beiseitezuschieben: Die Identifizierung wird nicht nur weitergetrieben wie bisher, sondern es wird die charakteristische Handlungsweise der Person formuliert, und durch die für die Beteiligten typische Formulierungsweise wird die implizite Zuordnung zu einer sozialen Kategorie angezeigt. ${ }^{14}$ Explizit hieße die Charakterisierung etwa folgendermaßen: Frau Xaver gehört zu den Personen, die regelmäßig zu allen Institutionen/Vereinen gehen, zu der sie als Angehörige der sozialen Kategorie 'Senioren' Zugang haben, dann, und nur dann, wenn es dort unentgeltliche Angebote gibt, vor allem Speise- und Getränkeangebote. Die Art der Institution deutet Frau Zimmermann in ihrem anschließenden Beleg an: hot de parre wa"s >is =se aa dort. parre ( = Pfarrer) referiert auf die Seniorennachmittage, die einmal wöchentlich von der evangelischen Gemeinde als Bibel- und Kaffeestunden abgehalten werden.

Auffallend an dieser Stelle der zentralen Charakterisierung und Kategorisierung des Tratschobjekts ist die Modalität, in der die Beteiligten das 'Skandalon' bearbeiten. Weder prosodisch noch verbal wird Entrüstung, moralische Verurteilung o.ä. gezeigt: Die Prosodie vermittelt nur den Eindruck von Bedeutsamkeit, explizite Be- oder Verurteilungen fehlen, wie das sonst im Tratsch an zentralen Stellen der Fall ist, an denen an der Kategorisierung des Tratschobjekts gearbeitet wird. Dort treten dann Mißbilligungsformeln auf, wie: sowas macht ma doch net oder moralische Beurteilungen wie: des is doch eine un-

14 Die gemeinsam vorgenommene Charakterisierung zeigt folgende, für die Formulierung von kategoriengebundenen Handlungsweisen typische Merkmale (vgl. W. Kallmeyer/ I. Keim (1989b):

- apodiktische Generalisierung: keine Verwendung von Quantoren, wie manchmal, öfter, immer o.ä.;

- Entindexikalisierung: keine Angaben zu Ort und Zeit;

- semantische Aussparung: das unbestimmte Lokaladverb iwwerall als Ortsbezeichnung wird nur durch einen semantisch leeren Relativsatz bestimmt, der aus Relativadverb wo, Personalpronomen es, Indefinitpronomen was und dem semantisch unspezifischen Verb geben besteht. Alle Pro-Formen sind vor dem Hintergrund der Definitionselemente der sozialen Kategorie semantisch aufzufüllen, auf die sie referieren, nämlich auf die absahnerin. 
verschämtheit. Außerdem treten dort kategoriale Benennungen auf, die im vorliegenden Beispiel fehlen. Auch die Reaktion der Beteiligten zeigt hier keine Entrüstung o.ä., sondern sie lachen bei der Zuordnung des Tratschobjekts zu einer sozialen Kategorie, die von allen Beteiligten an anderen Stellen im Material vehement verurteilt wird. Durch diese unübliche Bearbeitung eines Skandalons (implizite Zuordnung zu einer negativen sozialen Kategorie, Fehlen von Entrüstung und moralischer Verurteilung, 'bedeutsame' Darstellung und verstehendes Lachen der Rezipientinnen) markieren die Beteiligten, da $\beta$ es sich hier nicht um einen 'normalen Tratsch' handelt, bei dem das Objekt abwesend ist, drastisch und schonungslos beurteilt und explizit kategorisiert wird, sondern daß eine milde, schonende Form praktiziert wird. Diese besondere Bearbeitungsform deutet auf eine 'zweite Lesart' des Tratsches: Es geht hier nicht nur um die abwesende Frau Xaver, sondern vor allem um die anwesende Frau Kunz.

Wiederum ist Frau Kunz die einzige der Beteiligten, die auf die indirekte Charakterisierung von Frau Xaver reagiert; verbal und prosodisch drückt sie moralische Verurteilung aus und distanziert sich durch Kontrastierung eigener Handlungsprojektion mit der Handlung von Frau Xaver: nää sowas ded =sch gar net mache. Etwas später stellt sie explizit dar, daß sie sich Kuchen und Kaffee selbst kaufen würde.

Als einzige der Anwesenden mißbilligt Frau Kunz Frau Xavers Verhalten und stellt ihre eigene (zwar konjunktivisch und futurisch formulierte) Handlungsweise als der von Frau Xaver diametral entgegengesetzt dar. An dieser Stelle erfüllt sie zwei Aufgaben:

- Sie liefert die von den anderen Beteiligten ausgesparten, aber für die 'Normalform' des Tratsches konstitutiven Elemente der moralischen Bewertung und kooperiert damit entscheidend bei der Herstellung der ablaufenden Interaktion als Tratsch.

- Die Lücke, die ihr die anderen Beteiligten offenließen durch Aussparung eines wesentlichen Tratschelements, nutzt Frau Kunz zur expliziten Distanzierung von Frau Xavers Handlungsweise und zur Darstellung präferierter eigener Handlungsweisen, die in Übereinstimmung mit allgemeinen Gruppennormen stehen.

Frau Kunz zeigt hiermit, da $\beta$ sie das Anliegen der anderen auf der 'zweiten Ebene' verstanden hat, denn sie kommt mit ihrer Reaktionsweise zumindest auf der kommunikativen Oberfläche den Erwartungen der anderen entgegen, indem sie sich von ihrem skandalösen Verhalten lossagt.

In Gegenwart von Frau Kunz wurde in der Gruppe vor diesem Tratschereignis noch nicht die an ihr kritisierte Verhaltensweise offen thematisiert, deshalb erfolgt auch hier wieder Kritik in indirekter Form. Frau Kunz weiß seit einiger Zeit von dieser Kritik und hat sich bereits erfolgreich dagegen gewehrt. In harten Frotzelangriffen gelang es den Kritikerinnen nicht, sie betroffen oder gar einsichtig zu machen. Frau Kunz reagierte mit Unverfrorenheit und ließ die Kritikerinnen abblitzen. Als Steigerungsform für indirekte Kritik verwenden die Kritikerinnen hier den Tratsch und sind in der Situation erfolgreich: Frau Kunz hat die Kritik insoweit angenommen, als sie sich explizit von dem kritisierten Verhalten distanziert. Ihr Verhalten jedoch ändert sie in der Folge nicht. Es folgt offene Kritik, kurze Zeit später scheidet Frau Kunz aus der Gruppe aus. 


\subsubsection{LITERATURGRUPPE: TRATSCH ÜBER GRUPPENEXTERNE}

Vergleichbare Konfliktanlässe wie das 'Absahnen' gibt es in der Literaturgruppe nicht. Eher mokieren sich die Frauen über ein Zuviel des Angebots einer Gastgeberin, weil es mit einem Perfektionsanspruch verbunden sein könnte, dem sie sich nicht unterwerfen wollen. In der Literaturgruppe entfallen überhaupt Konflikte, die man 'gruppensprengend' nennen könnte, jedenfalls in den aufgenommenen Sitzungen. Das liegt u.a. daran, daß die Mitglieder danach ausgesucht wurden, ob sie zu den Frauen paßten, die die Gruppe initiierten. Eine Frau mit großen persönlichen Problemen zum Beispiel, von der die Frauen befürchteten, sie könnte damit die Gruppe zu sehr belasten, wurde nicht aufgenommen.

Am ehesten könnte ein Verhalten gruppensprengend wirken, das die persönliche Sphäre einer Beteiligten verletzt, sei es, daß sie sich in einem wesentlichen Aspekt ihres Selbstverständnisses angegriffen fühlt, sei es, $\mathrm{da} \beta$ eine Beteiligte zu selbstbezogen ist und die Aufmerksamkeit und das Mitgefühl der anderen zu stark an sich bindet. Die Frauen klatschen über solche aufdringlichen Leute, aber der Klatsch ist auf Gruppenexterne beschränkt. Es wird auch nicht - wie in der Bastelgruppe - über bestimmte Eigenschaften und Verhaltensweisen mit der Absicht geklatscht, daß der Adressat die Kritik dem Klatschobjekt zuträgt.

In den Aufnahmen gibt es aber eine interessante Passage, wo eine Frau aus dem gemeinsamen Gespräch ausbricht und einer anderen von einer Frau erzählt, die ununterbrochen sprechend anderen Leuten ihr Leid klagt und diese nicht zu Wort kommen läßt. Dies geschieht genau zu dem Zeitpunkt, als eine Frau der Gruppe ebenfalls ihr Leid klagt, aber in einer Weise, die nicht zu den für die Gruppe üblichen Behandlungsweisen führt, nämlich Anteilnahme zu zeigen und Lösungen zu planen. In mehreren Anläufen stellt diese Frau ihr Problem dar, aber die Zuhörerinnen sind ratlos, weil sie nicht verstehen, warum der geschilderte Vorfall die Sprecherin überhaupt bekümmert; sie sehen das Problem ganz woanders. Zweimal erklärt hier eine Zuhörerin, daß sie das auslösende Ereignis als nicht schlimm ansieht und daß die betroffene Frau es ignorieren solle. Das Unverständnis für ihr Leiden führt die Sprecherin dazu, immer klagender und eindringlicher zu sprechen. An dieser Stelle berichtet also eine der Zuhörerinnen vom aufdringlichen Verhalten einer externen Frau in einer Freizeitgruppe:

1 ST: du wei"ßt doch si"cher daß ich bei der frau Mayer in einem

2 ST: X-kurs bin (... ... ... ... hab ich gesagt) tdaß mich das so"

3 ST: fu"rchtbar nervt $\rightarrow$ und ich weiß ni"cht wie" ich das ** wie" ich

4 ST: das $\star \star$ ändern soll $\downarrow \star \star[\ldots]$ da i"s eine (...dame) dabei $\downarrow$ auch

5 ST: eine \#Y-inı\# die macht die tür auf $\uparrow$ * und erzählt von ihren K: \# BERUF \#

6 ST: Z-problemen $\downarrow$ * und erzählt während der ga"nzen zwei" stu"nden

7 ST: über die"se sa"che und über ihr ki"nd u"nd u"nd u"nd * und ich

8 ST: krieg kau"m luft (kann mich nicht) konzentrieren und geh so:" * 
9 ST: \#zerri"ssen\# und \#zerrü"ttet\# aus die"sen zwei stu"nden raus- *

$K$ : \# SEHR BETONT \# \# SEHR BETONT \#

10 ST: und ka"nn das ja aber praktisch gar nich der frau Mayer sagen

11 ST: denn $[\ldots]$

Das Verhalten der Frau in der Tratschgeschichte entspricht dem Verhalten der anwesenden Leidklagenden darin, daß sie auf ihrem Thema über Gebühr insistiert, nicht jedoch darin, $d a ß$ sie die anderen nicht zu Wort kommen läßt. Diese teilweise Parallelität des Verhaltens bleibt aber unausgesprochen und möglicherweise unbewußt. In Tratschgeschichten über Externe spießen die Frauen einerseits rücksichtsloses, aufdringliches Verhalten auf, andererseits Unwissenheit und Planlosigkeit in Dingen, die für sie Routineangelegenheiten sind. Aufdringliches Gesprächsverhalten würde eine wesentliche Gruppennorm verletzen. Die Frauen nehmen Rücksicht darauf, nicht zu lange das alleinige Sprecherrecht zu behalten. Auch bei sehr ausgedehnten Problemdarstellungen lassen sie den anderen Raum, sich am Thema zu beteiligen. Sie sprechen selten gleichzeitig, um das Wort zu behalten (im Gegensatz zum Simultansprechen zum Ausdruck von Konsenses), und sie lassen von eigenen Gesprächsinitiativen ab, wenn sie sehen, da $\beta$ eine andere aufgenommen wird.

\section{STATT EINES VERGLEICHS - GEGENÜBERSTELLUNG STILISTISCHER MERKMALE DER KONFLIKTBEARBEITUNG IN BEIDEN GRUPPEN}

Ein Vergleich der kommunikativen Stile von Angehörigen unterschiedlicher sozialer Welten setzt die Beschreibung der jeweiligen kulturellen Systeme voraus. Wir haben hier nur Ausschnitte aus den jeweiligen Systemen vorgestellt, die bezüglich der jeweils anstehenden kommunikativen Aufgaben nicht direkt vergleichbar sind. Auch die beobachteten Konfliktanlässe haben für die Gruppen einen anderen Stellenwert. Ganz allgemein lassen sich bei der Manifestation von unterschiedlich schweren Konflikten gegenläufige Bearbeitungstendenzen in den Gruppen feststellen: Mit zunehmender Schwere führen die Konfliktanlässe zu immer variantenreicheren Formen der Auseinandersetzung in der Bastelgruppe; in der Literaturgruppe dagegen werden schwerwiegende Konfliktpotentiale entweder umgangen oder sehr behutsam und andeutend behandelt.

Was wir darüber hinaus hier leisten können, ist die Zusammenfassung der jeweiligen Bearbeitungsformen und Durchführungsweisen eines bestimmten Konfliktanlasses für eine Gruppe und im Kontrast dazu die Entsprechung bzw. Markierung der Leerstelle für die andere Gruppe.

Konflikte, die aus einer Störung des Interaktionsziels entstehen, sind in der Bastelgruppe eher marginal, da in der Gruppe über das vorrangige Ziel der Treffen ein Konsens besteht. Konflikte dieser Art werden in der Regel schnell und problemlos durch formel- 
haften Austausch bearbeitet. Gelegentlich auftretende 'Rücksichtslosigkeiten' vor allem der Gruppenbetreuerin gegenüber (sie hat aufgrund ihrer Position öfter dem Geselligkeitsziel entgegenlaufende Interaktionsinteressen) werden nach kurzer Bearbeitung beigelegt.

Konflikte, die aus der Störung des Interaktionsziels entstehen, treten in der Literaturgruppe häufiger auf; sie ergeben sich strukturell aus dem Interessenwiderspruch der Beteiligten. Das offizielle Ziel der Gruppentreffen (Literaturlesung und Besprechung) steht in Konkurrenz zum inoffiziellen Zweck, dem Austausch und der Erörterung persönlicher Informationen und Probleme. Zur Bearbeitung dieses Konflikttyps haben die Frauen ein variantenreiches Repertoire entwickelt, das durch ironische Übertreibung, durch scherzhafte Bemerkungen, durch das Spiel mit Formeln oder durch das Ablenken vom Tadel die distanzwahrende Bearbeitung dieses Konflikts ermöglicht.

Tabuthemen betreffen aufgrund der unterschiedlichen Voraussetzungen der Gruppen ganz verschiedene Sachverhalte und haben in den Gruppen jeweils anderen Stellenwert. Die Mitglieder der Bastelgruppe dulden es nicht, daß persönliche Probleme, von denen alle wissen, zum Tabuthema gemacht werden. Wenn diese Norm verletzt wird, wird die Betroffene in indirekter Weise durch hartes Frotzeln bloßgestellt. Auf Imageverletzung erfolgt keine Reparatur. Persönliche Probleme in der Gruppe werden unter dem Aspekt der Geselligkeit präsentiert. Die ernste Bearbeitung von persönlichen Problemen ist in der Gruppe nicht erwünscht, sie findet nur unter besonders vertrauten Mitgliedern statt. Für die Bearbeitung dort gelten ganz andere Regeln. Versuche der ernsten Problembearbeitung als Gruppenaktivität gehören zu den Ausnahmen und werden von der Gruppe entweder ohne aktive Beteiligung der anderen kurzzeitig geduldet oder abschlägig behandelt.

In der Literaturgruppe gehört die Bearbeitung persönlicher Probleme in ernster Modalität zum inoffiziellen Zweck der Treffen. Die Betroffene bringt in der Regel ihr Problem selbst ein oder willigt in die Thematisierung durch andere ein. Bei schweren persönlichen Problemen wird eine gewisse Zurückhaltung der Betroffenen respektiert; aber sie wird dazu eingeladen, ihr Problem zu besprechen. Die Beteiligten bieten sich dann als solidarische Interpreten des Problems und als Helferinnen an.

Zur Bearbeitung schwerwiegender Konflikte, die Divergenzen bezüglich der Definition und Bewertung von Status- oder Rollenkategorien betreffen, werden in der Bastelgruppe mehrere Formen verwendet mit dem zunehmenden Zwang zur interaktiven Beteiligung der Kritisierten: hartes Frotzeln, indirekter Tratsch und Streit. Konflikte auf der Sachverhaltsebene werden dabei immer indirekt und andeutend bearbeitet. Harte Imageangriffe werden höchstens in der Formulierungs- und Sprechweise etwas abgeschwächt, aber nicht repariert. Im Streit werden Ärger und Aggression mit hoher Expressivität und durch Beschimpfungen ausgedrückt. Als Bestrafung für das konfliktauslösende Verhalten erfolgt die Falschkategorisierung und die Zuordnung zu einer negativ bewerteten sozialen Kategorie. Ziel aller Bearbeitungsformen für schwerwiegende Konflikte ist es, die Kritisierte zu treffen und bei ihr eine Verhaltensänderung zu bewirken. Dieser offensive und harte Umgang miteinander hängt u.a. auch mit den Zugangsbedingungen zur Gruppe zusammen. Nach Auffassung der Organisatoren des Vereins ist die Gruppe prinzipiell für alle Interessierten offen, d.h. es findet keine Vor- 
auswahl des Zugangs nach gruppeninternen Kriterien wie bei der Literaturgruppe statt. Gruppenkonstituierende Aktivitäten der Aushandlung von Normen und Wertorientierungen nehmen deshalb breiten Raum ein, ebenso wie Aktivitäten der allmählichen Eingliederung bzw. Ausgrenzung neuer Mitglieder.

In der Literaturgnuppe dagegen werden Differenzen mit dem Potential zu schwerwiegenderen Auseinandersetzungen nicht bearbeitet. Konflikte dieses Typs werden umgangen; gleichzeitig versuchen die Frauen partielle Gemeinsamkeiten herauszuarbeiten. Die soziale Kategorisierung betrifft vor allem familiäre Rollenkategorien, die Bearbeitung der Rollen als Ehefrau und Mutter. Wertunterschiede werden hier vor allem argumentativ besprochen.

Erhebliche Unterschiede zwischen den Gruppen sehen wir auch in der reflexiven Einstellung zur Konfliktbearbeitung, in dem Ausma $\beta$, in dem das eigene Sprechen kontrolliert wird und in dem über die anstehende Interaktion reflektiert wird. Die Frauen der Literaturgruppe thematisieren in höherem Maße die Möglichkeit bzw. Erlaubtheit, über Themen zu sprechen, die einen negativen Imagebezug zu einem Gruppenmitglied haben. Ebenso wird die Äußerung negativer Gefühle vermieden bzw. spontan geäußerte negative Wertungen werden wieder zurückgenommen. Für die Bastelgruppe dagegen ist bezeichnend, $d a ß$ negative Gefühle offen gezeigt und bearbeitet werden können.

Wir meinen, daß diese unterschiedlichen Befunde mit den unterschiedlichen Gruppenauffassungen über die Notwendigkeit oder Erlaubtheit kommunikativer Direktheit ebenso wie bei imagegefährdenden Aktivitäten zusammenhängen. Doch bevor wir hier weitergehende Aussagen machen können, müssen wir die Realisierungsweisen anderer kommunikativer Handlungen und Aufgaben mit in Betracht ziehen, um feststellen zu können, welche übergreifenden sozialstilistischen Kommunikationsformen es in beiden Gruppen gibt. 

ANHANG
Allgemeine Transkriptionszeichen
$=$
*
**
*3*
/
(...)
(ja)
aber ja
nein nie
jai
ja-
jaı
ja"
ja:
ja:
$<\mathrm{ja}$
$>$ ja
- manchmal
- manchmal
[...]
HEUTE

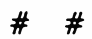
(wer?wem)
Verschleifung zwischen Wörtern bei Tilgung eines oder mehrerer Laute,
z.B. sa = mer ( = sag mir)
kurze Pause
längere Pause
längere Pause mit Angabe der Dauer in Sekunden
Wort- und Konstruktionsabbruch
unverständliche Sequenz
vermuteter Wortlaut
simultan gesprochene Sequenzen sind unterstrichen
Intonation steigend
Intonation schwebend
Intonation fallend
auffällige Betonung
auffällige Dehnung
sehr lange Dehnung
lauter im Vergleich zum direkt Vorhergehenden
leiser im Vergleich zum direkt Vorhergehenden
langsamer im Vergleich zum direkt Vorhergehenden
schneller im Vergleich zum direkt Vorhergehenden
Auslassung
Kommentar in Großbuchstaben in der Kommentarzeile;
Kommentarzeile ist dem Sprecher zugeordnet
Extension des Kommentars in Text- und Kommentarzeile
Alternativlautungen 


\section{TRANSLITERATION DER MANNHEIMER MUNDART}

Lautung, die weitgehend der Standardlautung entspricht, wird nach den geltenden Orthographieregeln wiedergegeben. Mundartliche Lautung wird graphematisch transliteriert. Im besonderen gilt:

1. Die für die Mannheimer Mundart allgemein geltende Nasalierung von Vokalen vor Nasalen wird nur in starker Ausprägung markiert. Starke Nasalierung, in der Regel verbunden mit Nasaltilgung, Verdunkelung und Längung des Vokals, wird durch "-" über dem Vokal angezeigt; z.B. std. /anziehen/ erscheint mdl. realisiert als äziehe bzw. öziehe.

2. Mundartliche Vokaldehnung, ob als Ergebnis des Monophthongierungs- oder des Konsonantentilgungsprozesses wird durch Vokaldoppelung translitiert. So kann std. /gesagt/ mdl. als [gsa:d] realisiert sein, das dann als gsaad transliteriert wird; ebenso wird std. /laufen/, mdl. als [la:fə] realisiert, durch laafe transliteriert. Unter dem Einfluß von Schnellsprechregeln bzw. besonderen Akzentuierungsregeln kann auch ein Kurzvokal auftreten, so daß z.B. std. /auch/ neben mdl. aach bzw. aa auch als $a$ transliteriert erscheint.

3. Mundartliche Entrundung des std. [y:] zu [i:] wird durch /ie/ transliteriert, z.B. std. /spülen/ als mdl. spiele bzw. schbiele.

4. Mundartliche Vokalkürzung wird durch Doppelung der Folgekonsonanten markiert; z.B. std. /geredet/, mdl. [gəR $\varepsilon_{t^{h}}{ }^{\mathrm{h}}$ ] wird als geredd transliteriert. Fällt zusammen mit Vokalkürzung vor Konsonantengruppen der dem Vokal direkt folgende Konsonant aus (vor allem $/ r /$ in der Kombination $/ r t /$ ), wird der nächste Konsonant gedoppelt. So erscheint std. /garten/ mdl. als gadde transliteriert, ebenso wie std. /warten/ als wadde.

5. Die allgemeine sprechsprachliche Tendenz zur Lenisierung von Fortes wird nur bei starker Lenisierung als Merkmal Mannheimer Mundart auch graphemisch wiedergegeben. So erscheint std. /steht/ bei standardnaher Artikulation transliteriert als steht und bei mundart-naher Artikulation als schded/t.

6. Wenn palatale Reibelaute [c] als dentale Reibelaute [ $[$ ], [६] ausgesprochen werden, werden sie als sch transliteriert also std. /ich/ als isch, ebenso wie std. /höflich/ als höflisch. 


\section{LITERATUR}

Bergmann, J.R.,1987. Klatsch. Zur Sozialform der diskreten Indiskretion. Berlin, New York: de Gruyter.

Brown, P., St. C. Levinson, 1987. Politeness: some universals in language use. Cambridge etc.: Cambridge University Press.

Goffman, E., 1971. Interaktionsrituale. Über Verhalten in direkter Kommunikation. Frankfurt/M.: Suhrkamp.

Kallmeyer, W., 1988. Wir und die anderen. Sprachliche Symbolisierung sozialer Identität und soziale Segmentierung. In: P. Nelde (ed.) 1989. Contact and Conflict. Bonn: Bouvier (In Vorbereitung).

- (Hg.) 1989a. Leben in der Stadt. Ethnographien von Mannheimer Stadtteilen. Mannheim (masch.).

- (Hg.) 1989b. Kommunikation in der Stadt. Exemplarische Analysen des Sprachverhaltens in Mannheim. Mannheim (masch.).

Kallmeyer, W./I. Keim 1986. Formulierungsweise, Kontextualisierung und soziale Identität. Dargestellt am Beispiel des formelhaften Sprechens. In: B. Schlieben-Lange (Hg.). 1986. Sprache und Wissen. Zeitschrift für Literaturwissenschaft und Linguistik (LiLi) 16. Heft 64, 98-126.

- 1989a. Formelhaftes Sprechen in der Filsbachwelt. In: W. Kallmeyer (Hg.). 1989b.

- 1989b. Bezeichnungen, Typisierung und soziale Kategorien. Untersucht am Beispiel der Ehe in der Filsbachwelt. In: W. Kallmeyer (Hg.). 1989b.

Keim, I., 1989. Ethnographie der Westlichen Unterstadt Mannheims. In: W. Kallmeyer (Hg.). 1989a.

Raible, W., 1987. Sprachliche Höflichkeit. Realisierungsformen im Deutschen und Französischen. Zeitschrift für französische Sprache und Literatur. Bd. XCVII. Heft 2, 1989, 145-168.

Rehbock, H., 1987. Konfliktaustragung in Wort und Spiel. Analyse eines Streitgesprächs von Grundschulkindern. In: G. Schank, J. Schwitalla (Hg.). Konflikte in Gesprächen. Tübingen: Narr, 176-138.

Schlieben-Lange, B., 1983. Traditionen des Sprechens. Elemente einer pragmatischen Sprachgeschichtsschreibung. Stuttgart: Kohlhammer.

Schwitalla, J., 1989. Ethnographie von Mannheim-Vogelstang. In: W. Kallmeyer (Hg.). 1989a. 